\title{
Gluon self-energy in a two-flavor color superconductor
}

\author{
Dirk H. Rischke \\ Institut für Theoretische Physik \\ Johann Wolfgang Goethe - Universität \\ Robert-Mayer-Str. 8-10, D-60054 Frankfurt/Main, Germany \\ email:drischke@th.physik.uni-frankfurt.de
}

\begin{abstract}
The energy and momentum dependence of the gluon self-energy is investigated in a color superconductor with two flavors of massless quarks. The presence of a color-superconducting quark-quark condensate modifies the gluon self-energy for energies which are of the order of the gap parameter. For gluon energies much larger than the gap, the self-energy assumes the form given by the standard hard-dense loop approximation. It is shown that this modification of the gluon self-energy does not affect the magnitude of the gap to leading and subleading order in the weak-coupling limit.
\end{abstract}

\section{INTRODUCTION}

Single-gluon exchange between two quarks is attractive in the color-antitriplet channel. Therefore, sufficiently cold and dense quark matter is a color superconductor [1]. When the quark-chemical potential $\mu \gg \Lambda_{\mathrm{QCD}}$, asymptotic freedom [2] implies that the strong coupling constant $g$ at the scale $\mu$ is much smaller than unity, $g(\mu) \ll 1$. This allows a controlled calculation of the color-superconducting gap parameter $\phi$ in the weak-coupling limit.

In order to compute the gap parameter, one has to solve a gap equation. In general, the gap equation determines the gap parameter $\phi(K)$ as a function of 4 -momentum $K^{\mu} \equiv\left(k_{0}, \mathbf{k}\right)$ of the fermionic quasiparticles. However, of physical interest is only the value of the gap function on the quasiparticle mass shell [3], i.e., for $k_{0} \equiv \epsilon_{k}$, where $\epsilon_{k} \equiv \sqrt{\left(\omega_{k}^{0}-\mu\right)^{2}+\phi^{2}}$ is the fermionic quasiparticle excitation energy. Here, $\omega_{k}^{0}$ is the kinetic energy of non-interacting particles, $\omega_{k}^{0}=k \equiv|\mathbf{k}|$ in the ultrarelativistic case. Then, at zero temperature, $T=0$, the gap equation is typically of the form

$$
\phi\left(\epsilon_{k}, \mathbf{k}\right)=g^{2} \rho(\mu) \int \frac{\mathrm{d} q}{\epsilon_{q}} \int \mathrm{d} \cos \theta \Delta\left(\epsilon_{k}-\epsilon_{q}, \mathbf{k}-\mathbf{q}\right) \phi\left(\epsilon_{q}, \mathbf{q}\right) .
$$

Here, $\rho(\mu)$ is the density of states at the Fermi surface, $\rho(\mu) \sim \mu^{2}$ in the ultrarelativistic case. Furthermore, it is assumed that the attractive interaction between fermions is mediated by boson exchange, with $g$ being the fermion-boson coupling constant and $\Delta(P)$ the boson propagator; $\theta \equiv \arccos (\mathbf{k}, \mathbf{q})$ is the angle between the 3-momenta of in- and out-going fermions in a scattering process.

For an ordinary superconductor, standard BCS theory [4,5] generally assumes that the attractive phononexchange interaction between electrons is local, i.e., a point-like four-fermion interaction, and isotropic, $\Delta(P) \equiv 1 / \Lambda^{2}$, where $\Lambda$ is a constant with the dimension of energy. As a consequence, the integral over cos $\theta$ in Eq. (1) is trivial. In a superconductor, the maximum phonon momentum is $p_{D}$, the Debye momentum. In weak coupling, $p_{D} \sim g \mu \ll \mu$. Consequently, the momentum exchange between electrons in a phononmediated scattering process is limited to the range $|\mathbf{k}-\mathbf{q}| \leq p_{D}$. Let us consider $k=k_{F}$, where $k_{F}$ is the Fermi momentum, and denote the value of the gap function at the Fermi surface by $\phi$. The restriction on possible phonon momenta then translates to limiting the $q$-integration to the (in weak coupling, narrow) region $k_{F}-p_{D} \leq q \leq k_{F}+p_{D}$ around the Fermi surface. Assuming that the momentum dependence of $\phi\left(\epsilon_{q}, \mathbf{q}\right)$ near the Fermi surface, $q \simeq k_{F}$, is weak, $\phi\left(\epsilon_{q}, \mathbf{q}\right) \simeq \phi=$ const., the gap equation simplifies to

$$
\phi=\frac{g^{2}}{c_{\mathrm{BCS}}} \ln \left(\frac{\omega_{D}+\sqrt{\omega_{D}^{2}+\phi^{2}}}{\phi}\right) \phi,
$$


where $1 / c_{\mathrm{BCS}} \sim \rho(\mu) / \Lambda^{2}$. Here, $\omega_{D} \equiv v_{F} p_{D}$ is the Debye frequency and $v_{F}$ the Fermi velocity, $v_{F}=1$ in the ultrarelativistic limit. For any, even arbitrarily weak, attractive interaction there is always a nontrivial solution $\phi \neq 0$ to this equation. Consequently, the constant factor $\phi$ common to both sides can be divided out. In weak coupling, $g^{2} \ll 1$, and in order to solve Eq. (2) the logarithm has to be $\sim 1 / g^{2} \gg 1$, to compensate the small prefactor $g^{2} \ll 1$. This requires $\phi \ll \omega_{D}$. Then one may approximate $\ln \left[\left(\omega_{D}+\sqrt{\omega_{D}^{2}+\phi^{2}}\right) / \phi\right] \simeq$ $\ln \left(2 \omega_{D} / \phi\right)$, and the solution is

$$
\phi=b_{\mathrm{BCS}} \mu \exp \left(-\frac{c_{\mathrm{BCS}}}{g^{2}}\right),
$$

where $b_{\mathrm{BCS}} \equiv 2 \omega_{D} / \mu$ is a dimensionless constant. This discussion shows that the constant $c_{\mathrm{BCS}}$ in the exponent is determined by the constant in front of the "BCS logarithm" $\int \mathrm{d} q / \epsilon_{q} \sim \ln \left(b_{\mathrm{BCS}} \mu / \phi\right)$, while the constant in the prefactor, $b_{\mathrm{BCS}}$, is determined by the constant "under" the BCS logarithm, in this case the size of the $q$-integration region.

The discussion can be easily generalized to non-local interactions of finite range, for instance massive scalar boson exchange [6]. In this case, $\Delta(P) \equiv\left(M_{B}^{2}-P^{2}\right)^{-1}$ is the boson propagator, with $M_{B}$ the boson mass, $P^{2} \equiv p_{0}^{2}-\mathbf{p}^{2}$. For the following, let us assume that fermions are massless, and that the boson mass is generated by in-medium effects, $M_{B} \simeq g \mu$, i.e., in weak coupling the different energy scales in the problem are ordered, $\phi \ll M_{B} \ll \mu$. The angular integral is no longer trivial; in the ultrarelativistic case and near the Fermi surface, $k \simeq q \simeq k_{F} \equiv \mu$,

$$
\rho(\mu) \Delta(P) \sim \frac{1}{2(1-\cos \theta)+M_{B}^{2} / \mu^{2}},
$$

where the energy dependence of the boson propagator has been neglected, since $p_{0} \equiv \epsilon_{k}-\epsilon_{q} \sim \phi \ll M_{B}$. Obviously, the term (位) enhances the contribution from small-angle fermion-fermion scattering, $\cos \theta \simeq 1$, to the gap equation (11). Performing the angular integral introduces an additional logarithm, in the following called "collinear logarithm", in comparison to Eq. (2),

$$
\phi=\frac{g^{2}}{c_{\mathrm{B}}} \ln \left(\frac{b_{\mathrm{B}} \mu}{\phi}\right) \ln \left(\frac{2 \mu}{M_{B}}\right) \phi,
$$

where I exploited the hierarchy of scales in weak coupling. The solution is rather similar to the one in BCS theory, Eq. (3),

$$
\phi=b_{\mathrm{B}} \mu \exp \left(-\frac{c_{\mathrm{B}}}{g^{2} \ln \left(2 \mu / M_{B}\right)}\right) .
$$

Quantitatively, the difference to Eq. (3) is that, in weak coupling, the gap becomes larger, since the coupling constant $g^{2}$ is replaced by $g^{2} \ln \left(2 \mu / M_{B}\right) \sim g^{2} \ln (1 / g) \gg g^{2}$.

In QCD, gluon exchange is also a non-local interaction. The difference to the previous case is that gluons are massless and thus the interaction has infinite range. In the vacuum, this holds for electric as well as magnetic gluons. In a dense medium, however, electric and non-static magnetic gluon exchange is screened. Essentially, these gluons behave like the scalar bosons of the previous example with $M_{B} \equiv m_{g}$, where

$$
m_{g}=\sqrt{\frac{N_{f}}{6 \pi^{2}}} g \mu
$$

is the gluon mass at $T=0$, with $N_{f}$ being the number of massless quark flavors participating in screening electric and non-static magnetic gluons. Screening reduces the range of the interaction to distances $<m_{g}^{-1}$. On the other hand, the exchange of almost static magnetic gluons is still unscreened and thus of infinite range.

In the vacuum, the gluon propagator is $\Delta(P) \sim-1 / P^{2}$, and the previously discussed collinear enhancement in the gap equation is much stronger: instead of being cut off by a mass term $M_{B}$, it is cut off by the gluon energy, $p_{0}=\epsilon_{k}-\epsilon_{q} \sim \phi \ll M_{B} \simeq g \mu$. (In the previous case, the boson energy was negligible compared to its mass.) The collinear logarithm in the gap equation is now $\sim \ln \left[2 \mu /\left|\epsilon_{k}-\epsilon_{q}\right|\right] \sim \ln (2 \mu / \phi)$. It is thus of the same order as the BCS logarithm, and consequently much larger than the collinear logarithm $\ln \left(2 \mu / M_{B}\right) \sim \ln (1 / g)$ in the previous case. In a dense medium, the gluon propagator is more complicated, 
but since the exchange of almost static magnetic gluons is unscreened, it still gives rise to the same collinear logarithm $\ln (2 \mu / \phi)$ as in the vacuum [3].

In QCD, there is no restriction on the magnitude of the gluon momentum in the gap equation. Nevertheless, in weak coupling the gap function $\phi\left(\epsilon_{q}, \mathbf{q}\right)$ is strongly peaked at the Fermi surface $q=k_{F}$ [3,7,8,8]. Effectively, this again restricts the $q$-integration to a narrow range $k_{F}-\delta \leq q \leq k_{F}+\delta$ around the Fermi surface, $\phi \ll \delta \ll \mu$. For an order-of-magnitude estimate (but not for a numerically correct solution), in this range one may take $\phi\left(\epsilon_{k}, \mathbf{k}\right) \simeq \phi\left(\epsilon_{q}, \mathbf{q}\right) \simeq \phi=$ const., such that the gap equation assumes the form

$$
\phi=\frac{g^{2}}{c^{2}}\left[\ln ^{2}\left(\frac{2 \delta}{\phi}\right)+b^{\prime} \ln \left(\frac{2 \delta}{\phi}\right)+d\right] \phi .
$$

The first term contains two powers of the logarithm $\ln (2 \delta / \phi)$. The first one is the BCS logarithm. The second one is the collinear logarithm arising from the exchange of massless gluons. (Any constant under this logarithm different from $2 \delta$ has been absorbed in $b^{\prime}$.) The value of the constant $c^{2}$ is determined by how many gluon degrees of freedom are unscreened and thus give rise to a collinear logarithm in addition to the BCS logarithm. In vacuum, these are all gluons, while in medium, only almost static magnetic gluons contribute to $c^{2}$. The second term contains only one power of the logarithm $\ln (2 \delta / \phi)$ : the BCS logarithm. In a strongly interacting, dense medium, this logarithm arises from exchange of screened electric and non-static magnetic gluons [3]. The value of $b^{\prime}$ is determined by the number of degrees of freedom of such gluons. As seen above, screened boson exchange also gives rise to a collinear logarithm, but this time of the form $\ln \left(2 \mu / M_{B}\right), M_{B} \equiv m_{g}$ for gluons. This logarithm has been absorbed in the constant $b^{\prime}$. Finally, all contributions without any logarithm have been summed up into the constant $d$.

In weak coupling, $\phi \ll \delta$ and $\ln (2 \delta / \phi) \gg 1$. Thus, all unscreened gluons contribute to leading order to the gap equation, i.e., to the first term in Eq. (8), while all screened gluons contribute to subleading order, i.e., to the second term. All other contributions make up the third, sub-subleading order term.

The solution of Eq. (8) is straightforward,

$$
\phi=b \mu \exp \left(-\frac{c}{g}\right)[1+O(g)],
$$

where $b \mu \equiv 2 \delta \exp \left(b^{\prime} / 2\right)$. A more thorough analysis 3 shows that $b$ is actually independent of $\delta$, and solely determined by $b^{\prime}$, i.e., by the contributions of screened gluons and the constants under the collinear logarithm. The constant $d$ does not appear to leading and subleading order in the solution of the gap equation; it is part of the terms of order $O(g)$ on the right-hand side of (9).

Observe that the power of the coupling $g$ in the exponent is reduced as compared to the BCS result (3) or the result (6) for massive boson exchange. This was first noted by Barrois 9, but never made it into the published literature. Barrois assumed that the gluon propagator is the same as in vacuum and consequently, as discussed above, all gluon degrees of freedom are unscreened.

In a dense medium, however, only almost static magnetic gluons are unscreened, while electric and nonstatic magnetic gluons are screened. In a seminal paper, Son [7] derived the result (9) with the gluon propagator in the so-called "hard-dense-loop" (HDL) limit 10,11, and obtained

$$
c=\frac{3 \pi^{2}}{\sqrt{2}} .
$$

Son also gave an estimate for the constant $b$,

$$
b=\frac{b_{0}}{g^{5}}
$$

with some constant $b_{0}$ of order one. The parametric dependence of $b$ on $g$ arises from the gluon mass, Eq. (7). Several authors [3, 8, 12, 13 have confirmed the results (10) and (11) and refined the estimate for $b_{0}$,

$$
b_{0}=512 \pi^{4}\left(\frac{2}{N_{f}}\right)^{5 / 2} b_{0}^{\prime}
$$

where the dependence on $N_{f}$ arises from that of the gluon mass (7), and $b_{0}^{\prime}=1$ under the present approximations. 
In a dense medium, not only the gluon propagator is modified. Brown, Liu, and Ren [14 included a finite, $\mu$-dependent contribution to the quark wavefunction renormalization in their estimate for $b_{0}^{\prime}$,

$$
b_{0}^{\prime}=\exp \left(-\frac{\pi^{2}+4}{8}\right) b_{0}^{\prime \prime} \simeq 0.176 b_{0}^{\prime \prime} .
$$

They also asserted that there are no further corrections to $b_{0}^{\prime}$ at this order in $g$, i.e., $b_{0}^{\prime \prime}=1+O(g)$.

Beane, Bedaque, and Savage [15] argued that the coupling constant in the gap equation should not be taken at the scale $\mu$, but at the scale of the momentum of the exchanged gluon. Using the standard running of the coupling constant in the vacuum they obtained $b_{0}^{\prime \prime}=\exp \left[33\left(\pi^{2}-4\right) / 64\right] \simeq 20$. At present, it remains an interesting open problem to check the validity of their arguments by systematically computing vertex corrections to the gap equation. Furthermore, their estimate could be improved replacing the vacuum running of the coupling by that in a dense medium.

Sub-subleading contributions, i.e., of order $g$ in the constant $b_{0}^{\prime \prime}$, or equivalently, contributions to the constant $d$ in Eq. (8), can arise from a variety of effects, for instance from the finite lifetime of quark excitations away from the Fermi surface [16]. Also, an apparent gauge dependence of the result (9) surfaces at this order 17]. However, the gap on the quasiparticle mass shell is a physical observable and thus in principle gauge-independent. This indicates that, for a complete solution to sub-subleading order, one has to go beyond the one-boson exchange approximation in the gap equation. This task appears, at least at present, too difficult to be feasible.

In this paper, I focus instead on a contribution which is potentially of leading or subleading order. At small temperatures $T \sim \phi \ll \mu$, the dominant contribution to the one-loop gluon self-energy comes from a quark loop; it is $\sim g^{2} \mu^{2}$, while gluon (and ghost) loops contribute a term $\sim g^{2} T^{2}$ and are thus suppressed [18]. In the standard HDL approximation, however, the quark excitations in the quark loop are taken to be those in a normal and not a superconducting medium. This is in principle inconsistent, as the fermion excitation spectrum in a superconductor differs from that in a normal conductor [6]. In Refs. [18,19] I argued that using the correct gluon self-energy in the gap equation could in principle affect the value of the color-superconducting gap [20]. Consequently, I derived general expressions for the gluon self-energy in the two- and three-flavor case, and computed the self-energy in the limit of vanishing energy and momentum, which yields the Debye and Meissner masses in a color-superconductor.

It turned out that in the two-flavor case, where the color-superconducting condensate breaks the $S U(3)_{c}$ color gauge symmetry to $S U(2)_{c}$, the three gluons corresponding to the unbroken $S U(2)_{c}$ subgroup remain massless, like in the vacuum [18]. Naively, if the propagator of these gluons is the same as in vacuum also at nonzero energy $p_{0}$ and momentum $\mathbf{p}$, this would change the number of modes mediating long-range interactions. Remembering the above discussion of the gap equation (8), this would affect the leading-order contribution to the gap equation and could in principle change the value (10) for the coefficient $c$.

It turns out, however, that for zero gluon energy $p_{0}=0$ and gluon momenta $|\mathbf{p}| \equiv p \gg \phi$, the self-energies approach their HDL form [18]. Moreover, for energies and momenta much smaller than the gap, $p_{0}, p \ll \phi$, these gluons do not behave exactly like in vacuum, but like in a medium with a large dielectric constant [21]. A change of the leading-order contribution to the gap equation, and thus of the coefficient $c$, appears therefore unlikely, but a change of the subleading contribution cannot be excluded. In order to decide this question, however, knowledge of the gluon self-energy in the limited range of energies and momenta $p_{0}, p \ll \phi$, or for $p_{0}=0$ and $p \gg \phi$ is insufficient; one has to compute the gluon self-energy for energies and momenta of relevance for the gap equation (11). As the integral on the right-hand side of (11) is dominated by quark energies and momenta close to the Fermi surface, the range of relevant gluon energies and momenta is $p_{0}, p \ll \mu$, but not the more restrictive range $p_{0}, p \ll \phi$ or the special case $p_{0}=0, p \gg \phi$. In the present paper, I therefore compute the gluon self-energy for $p_{0}, p \ll \mu$ in a two-flavor color superconductor.

The gap equation in the two-flavor case was derived in [3]. Assuming that Cooper pairs are formed from quarks with fundamental colors 1 and 2, such that the condensate has fundamental color (anti-) 3, one obtains

$$
\Phi^{+}(K)=\frac{3}{4} g^{2} \frac{T}{V} \sum_{Q} \gamma_{\mu}\left[\Delta_{11}^{\mu \nu}(K-Q)-\frac{1}{9} \Delta_{88}^{\mu \nu}(K-Q)\right] \Xi^{+}(Q) \gamma_{\nu}
$$

Here, $\Phi^{+}$is the gap matrix in Dirac space, $V$ is the volume of the system, $\gamma_{\mu}$ are Dirac matrices, $\Delta_{a b}^{\mu \nu}$ is the gluon propagator, and $\Xi^{+}$is the off-diagonal element of the quasiparticle propagator in Nambu-Gor'kov space. 
Only gluons mediating between gapped quarks enter the gap equation, i.e., gluons with adjoint colors 1 , 2, 3, and 8. Gluons mediating between gapped and ungapped quarks, i.e., gluons with adjoint colors 4, 5, 6, and 7, do not appear. Moreover, the propagators for gluons of the unbroken $S U(2)_{c}$ subgroup are identical, $\Delta_{11}^{\mu \nu}=\Delta_{22}^{\mu \nu}=\Delta_{33}^{\mu \nu}$, but different from the propagator for the eighth gluon, $\Delta_{88}^{\mu \nu}$.

I work in pure Coulomb gauge, where the gluon propagator for colors 1, 2, 3, and 8 assumes the form [18,22,

$$
\begin{aligned}
& \Delta_{a b}^{00}(P)=-\delta_{a b} \frac{1}{\mathbf{p}^{2}-\Pi_{a a}^{00}(P)} \\
& \Delta_{a b}^{0 i}(P)=0 \\
& \Delta_{a b}^{i j}(P)=-\delta_{a b}\left(\delta^{i j}-\hat{p}^{i} \hat{p}^{j}\right) \frac{1}{P^{2}-\Pi_{a a}^{t}(P)} .
\end{aligned}
$$

Here, $\Pi_{a a}^{00}(P)$ is the 00-component and

$$
\Pi_{a a}^{t}(P) \equiv \frac{1}{2}\left(\delta_{i j}-\hat{p}_{i} \hat{p}_{j}\right) \Pi_{a a}^{i j}(P)
$$

the spatially transverse component of the gluon self-energy $\Pi_{a a}^{\mu \nu}(P), \hat{\mathbf{p}} \equiv \mathbf{p} / p$. In the following, I will (somewhat imprecisely) term $\Pi_{a a}^{00}$ the electric self-energy, and $\Pi_{a a}^{t}$ the magnetic self-energy.

Apparently, in order to settle the question whether the modification of the gluon self-energy in a color superconductor (as compared to the HDL approximation) changes the solution of the gap equation (14), one only has to consider the following four components of the gluon self-energy: $\Pi_{11}^{00}, \Pi_{88}^{00}, \Pi_{11}^{t}$, and $\Pi_{88}^{t}$. The imaginary and real parts of these four components are computed in Sec. III. In Sec. III, I determine the spectral densities of electric and magnetic gluons as a function of gluon energy and momentum. Section IV discusses the effect of the modification of the gluon self-energy in a color superconductor on the solution of the gap equation. I conclude in Sec. V with a summary of the results. Unless mentioned otherwise, throughout this paper I work at zero temperature, $T=0$. I use natural units and the metric tensor $g^{\mu \nu}=\operatorname{diag}(+,-,-,-)$.

\section{THE GLUON SELF-ENERGIES}

The starting point of the computation of the electric and magnetic self-energies for gluons of color 1 are Eqs. (99a) and (99c) of [18]. For $N_{f}=2$ flavors,

$$
\begin{aligned}
\Pi_{11}^{00}(P)= & -\frac{1}{2} g^{2} \int \frac{\mathrm{d}^{3} \mathbf{k}}{(2 \pi)^{3}} \sum_{e_{1}, e_{2}= \pm}\left(1+e_{1} e_{2} \hat{\mathbf{k}}_{1} \cdot \hat{\mathbf{k}}_{2}\right) \\
& \times\left(\frac{1}{p_{0}+\epsilon_{1}+\epsilon_{2}+i \eta}-\frac{1}{p_{0}-\epsilon_{1}-\epsilon_{2}+i \eta}\right) \frac{\epsilon_{1} \epsilon_{2}-\xi_{1} \xi_{2}-\phi_{1} \phi_{2}}{2 \epsilon_{1} \epsilon_{2}}, \\
\Pi_{11}^{t}(P)= & -\frac{1}{2} g^{2} \int \frac{\mathrm{d}^{3} \mathbf{k}}{(2 \pi)^{3}} \sum_{e_{1}, e_{2}= \pm}\left(1-e_{1} e_{2} \hat{\mathbf{k}}_{1} \cdot \hat{\mathbf{p}} \hat{\mathbf{k}}_{2} \cdot \hat{\mathbf{p}}\right) \\
& \times\left(\frac{1}{p_{0}+\epsilon_{1}+\epsilon_{2}+i \eta}-\frac{1}{p_{0}-\epsilon_{1}-\epsilon_{2}+i \eta}\right) \frac{\epsilon_{1} \epsilon_{2}-\xi_{1} \xi_{2}+\phi_{1} \phi_{2}}{2 \epsilon_{1} \epsilon_{2}} .
\end{aligned}
$$

Here, $\mathbf{k}_{1} \equiv \mathbf{k}+\mathbf{p} / 2$ and $\mathbf{k}_{2} \equiv \mathbf{k}-\mathbf{p} / 2, \phi_{i} \equiv \phi_{\mathbf{k}_{i}}^{e_{i}}$ is the gap function for quasiparticles $\left(e_{i}=+1\right)$ or quasiantiparticles $\left(e_{i}=-1\right)$ with momentum $\mathbf{k}_{i}$, the variable $\xi_{i}$ is defined as $\xi_{i} \equiv e_{i} k_{i}-\mu$, and $\epsilon_{i} \equiv \sqrt{\xi_{i}^{2}+\phi_{i}^{2}}$ is the quasiparticle excitation energy. The difference between Eqs. (17) and Eqs. (99) of [18] is that the analytic continuation to real gluon energies $p_{0}$ has been made explicit via the $i \eta$ prescription [5], and that there are no terms $\sim N_{i} \equiv\left[\exp \left(\epsilon_{i} / T\right)+1\right]^{-1}$, since $N_{i} \equiv 0$ at $T=0$. (Note that $\epsilon_{i} \geq \phi_{i}>0$ in a superconductor.)

According to Eq. (78c) of [18], the self-energy for gluons of color 8 is

$$
\Pi_{88}^{\mu \nu}(P)=\frac{2}{3} \Pi_{0}^{\mu \nu}(P)+\frac{1}{3} \tilde{\Pi}^{\mu \nu}(P)
$$

where $\Pi_{0}^{\mu \nu}$ is the standard HDL self-energy. Its components of relevance for the following are 10,18. 


$$
\begin{aligned}
\Pi_{0}^{00}(P) & \simeq-3 m_{g}^{2} \int \frac{\mathrm{d} \Omega}{4 \pi}\left(1-\frac{p_{0}}{p_{0}+\mathbf{p} \cdot \hat{\mathbf{k}}+i \eta}\right), \\
\Pi_{0}^{t}(P) & \simeq \frac{3}{2} m_{g}^{2} \int \frac{\mathrm{d} \Omega}{4 \pi}\left[1-(\hat{\mathbf{k}} \cdot \hat{\mathbf{p}})^{2}\right] \frac{p_{0}}{p_{0}+\mathbf{p} \cdot \hat{\mathbf{k}}+i \eta},
\end{aligned}
$$

where $m_{g}$ is the gluon mass (7). According to Eqs. (102) of [18], the electric and magnetic components of the self-energy $\tilde{\Pi}^{\mu \nu}$ are

$$
\begin{aligned}
\tilde{\Pi}^{00}(P)= & -\frac{1}{2} g^{2} \int \frac{\mathrm{d}^{3} \mathbf{k}}{(2 \pi)^{3}} \sum_{e_{1}, e_{2}= \pm}\left(1+e_{1} e_{2} \hat{\mathbf{k}}_{1} \cdot \hat{\mathbf{k}}_{2}\right) \\
& \times\left(\frac{1}{p_{0}+\epsilon_{1}+\epsilon_{2}+i \eta}-\frac{1}{p_{0}-\epsilon_{1}-\epsilon_{2}+i \eta}\right) \frac{\epsilon_{1} \epsilon_{2}-\xi_{1} \xi_{2}+\phi_{1} \phi_{2}}{2 \epsilon_{1} \epsilon_{2}} \\
\tilde{\Pi}^{t}(P)= & -\frac{1}{2} g^{2} \int \frac{\mathrm{d}^{3} \mathbf{k}}{(2 \pi)^{3}} \sum_{e_{1}, e_{2}= \pm}\left(1-e_{1} e_{2} \hat{\mathbf{k}}_{1} \cdot \hat{\mathbf{p}} \hat{\mathbf{k}}_{2} \cdot \hat{\mathbf{p}}\right) \\
& \times\left(\frac{1}{p_{0}+\epsilon_{1}+\epsilon_{2}+i \eta}-\frac{1}{p_{0}-\epsilon_{1}-\epsilon_{2}+i \eta}\right) \frac{\epsilon_{1} \epsilon_{2}-\xi_{1} \xi_{2}-\phi_{1} \phi_{2}}{2 \epsilon_{1} \epsilon_{2}} .
\end{aligned}
$$

The difference between Eqs. (17) and (20) is the relative sign in front of the term $\sim \phi_{1} \phi_{2}$.

In the following, I compute real and imaginary parts of Eqs. (17) and (20) separately, using the identity

$$
\frac{1}{x+i \eta} \equiv \mathcal{P} \frac{1}{x}-i \pi \delta(x)
$$

for the energy denominators in Eqs. (17) and (20), where $\mathcal{P}$ stands for the principal value prescription. I begin with the imaginary parts.

\section{A. Imaginary parts}

In the computation of the imaginary parts it is sufficient to consider gluon energies $p_{0} \geq 0$ as $\operatorname{Im} \Pi\left(-p_{0}, \mathbf{p}\right) \equiv-\operatorname{Im} \Pi\left(p_{0}, \mathbf{p}\right)$ for all self-energies (17), (19), (20), which is easy to prove using Eq. (21), and noting that $\delta(x)=\delta(-x)$. It is instructive to first consider the imaginary parts of the HDL self-energies. For electric and magnetic gluons one obtains the standard result [10,23]

$$
\begin{aligned}
\operatorname{Im} \Pi_{0}^{00}(P) & \simeq-\pi \frac{3}{2} m_{g}^{2} \frac{p_{0}}{p} \theta\left(p-p_{0}\right), \\
\operatorname{Im} \Pi_{0}^{t}(P) & \simeq-\pi \frac{3}{4} m_{g}^{2} \frac{p_{0}}{p}\left(1-\frac{p_{0}^{2}}{p^{2}}\right) \theta\left(p-p_{0}\right),
\end{aligned}
$$

corresponding to Landau damping for space-like gluons.

For $\operatorname{Im} \Pi_{11}^{00}(P)$ and $p_{0}>0$, the delta-function originating from the first energy denominator in Eq. (17a) has no support, since $\epsilon_{i} \geq \phi_{i}>0$. For the delta-function originating from the second energy denominator, use the fact that the interesting range of gluon energies in the gap equation (14) is $p_{0} \ll \mu$, as the quarks in the Cooper pair are close to the Fermi surface where $\epsilon_{i} \sim \phi_{i} \ll \mu$. Then, the only term in the sums over $e_{1}, e_{2}$ in Eq. (17a) that one has to keep is the one where $e_{1}=e_{2}=+1$; for $e_{i}=-1, \epsilon_{i} \simeq\left|k_{i}+\mu\right| \sim \mu$ is far from the Fermi surface and the delta-function has no support for $p_{0} \ll \mu$.

Furthermore, the fermion momentum $\mathbf{k}$ in the loop is close to the Fermi surface, $k \sim \mu$, while the interesting range of gluon momenta in the gap equation is $p \ll k$, which allows to approximate

$$
\begin{aligned}
\hat{\mathbf{k}}_{1} \cdot \hat{\mathbf{k}}_{2} & \simeq 1 \\
\xi_{1} & \simeq \xi+\frac{\mathbf{p} \cdot \hat{\mathbf{k}}}{2} \equiv \xi_{+}, \\
\xi_{2} & \simeq \xi-\frac{\mathbf{p} \cdot \hat{\mathbf{k}}}{2} \equiv \xi_{-},
\end{aligned}
$$


where $\xi \equiv k-\mu$. Finally, setting $\phi_{1} \simeq \phi_{2} \equiv \phi$, and defining $\epsilon_{ \pm} \equiv \sqrt{\xi_{ \pm}^{2}+\phi^{2}}$, one obtains

$$
\operatorname{Im} \Pi_{11}^{00}(P) \simeq-\pi g^{2} \int \frac{\mathrm{d}^{3} \mathbf{k}}{(2 \pi)^{3}} \delta\left(p_{0}-\epsilon_{+}-\epsilon_{-}\right) \frac{\epsilon_{+} \epsilon_{-}-\xi_{+} \xi_{-}-\phi^{2}}{2 \epsilon_{+} \epsilon_{-}}
$$

First, note that since $\epsilon_{+}+\epsilon_{-} \geq 2 \phi$, the delta-function has no support for $p_{0}<2 \phi$ : the imaginary part of the gluon self-energy vanishes below the threshold $2 \phi$ for quasiparticle-quasihole excitations. This is clearly different from the HDL self-energy which has an imaginary part for all gluon energies $0<p_{0} \leq p$, cf. Eq. (22a).

The integration over $\mathbf{k}$ is best done in spherical coordinates, choosing $\mathbf{p}=(0,0, p)$. Then, the integration over the polar angle $\varphi$ becomes trivial. Replacing the integration over azimuthal angle $\theta$ by $x \equiv \cos \theta$ and over $k$ by $\xi \equiv k-\mu$, one can use the delta-function to either perform the integration over $x$, or over $\xi$. Choosing the latter, for a given value of $x$ one finds that there are two roots of the argument of the delta-function, which differ by an overall sign,

$$
\xi^{*}(x)= \pm \frac{p_{0}}{2} \sqrt{1-\frac{4 \phi^{2}}{p_{0}^{2}-p^{2} x^{2}}}
$$

It is instructive to draw these solutions in the $(x, \xi)$-plane, cf. Fig. 1. In part (a) of this figure, corresponding to $p_{0}>E_{p}$, where

$$
E_{p} \equiv \sqrt{p^{2}+4 \phi^{2}}
$$

these two roots exist for all values of $x \in[-1,1]$, while in part (b), corresponding to $2 \phi \leq p_{0} \leq E_{p}$, they merge at $\xi=0$ and $x= \pm t p_{0} / p$, where

$$
t \equiv \sqrt{1-\frac{4 \phi^{2}}{p_{0}^{2}}}
$$

there is no solution for $1 \geq|x|>t p_{0} / p$. For $p_{0}<2 \phi$, there is no solution either, as already mentioned above. Note that $E_{p}=2 \phi$ for $p=0$, while $E_{p} \simeq p$ for $p \gg 2 \phi$. Analogously, $t=0$ for $p_{0}=2 \phi$, while $t \simeq 1$ for $p_{0} \gg 2 \phi$.

In the limit $\phi \rightarrow 0$, the $x$-integration decouples from the integration over $\xi$, and by Eq. (25), the value of the latter is simply constant, $\xi^{*} \equiv \pm p_{0} / 2$. This is indicated by the dashed lines in Fig. 11.

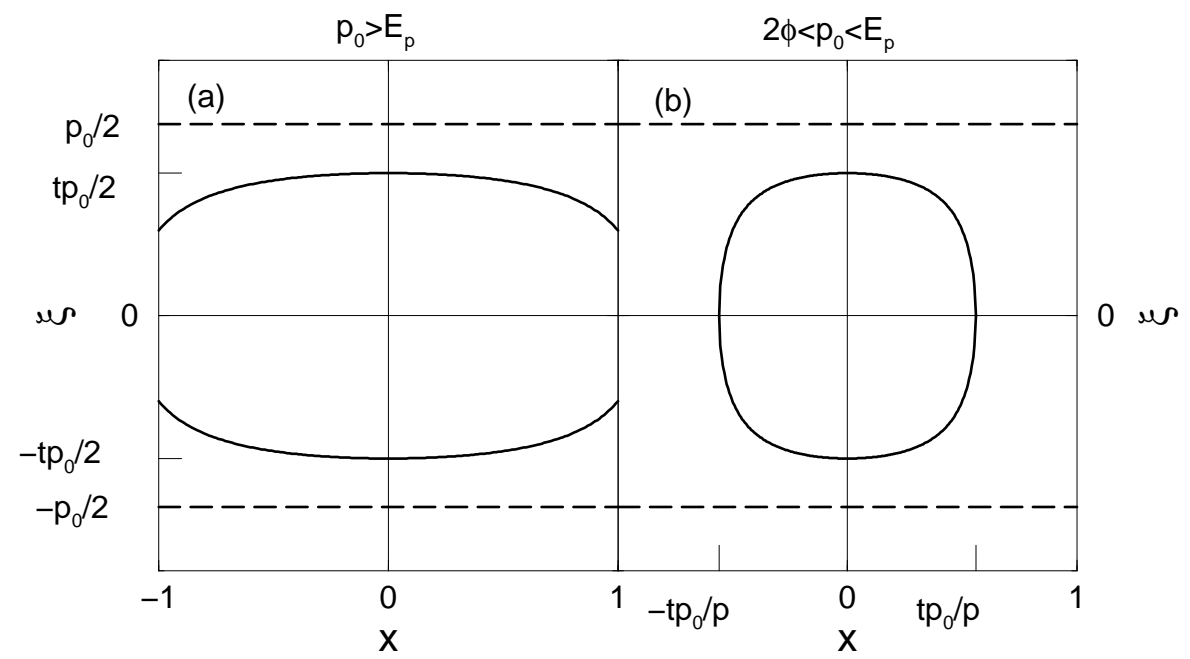

FIG. 1. Solid lines: the function $\xi^{*}(x)$ from Eq. (25) for (a) $p_{0}>E_{p}$, and (b) $2 \phi \leq p_{0} \leq E_{p}$. Dashed lines represent the case $\phi=0$. 
Evaluating the $\xi$-integral at the roots (25), one obtains

$$
\operatorname{Im} \Pi_{11}^{00}(P) \simeq-\pi \frac{3}{2} m_{g}^{2} \theta\left(p_{0}-2 \phi\right) \frac{4 \phi^{2}}{p_{0} p} \int_{0}^{u} \mathrm{~d} y \frac{y^{2}}{\left(1-y^{2}\right)^{3 / 2} \sqrt{t^{2}-y^{2}}},
$$

where $u \equiv \min \left(t, p / p_{0}\right)$. The integral can be expressed in terms of elliptic integrals, yielding the final answer

$$
\begin{aligned}
\operatorname{Im} \Pi_{11}^{00}(P) & \simeq-\pi \frac{3}{2} m_{g}^{2} \theta\left(p_{0}-2 \phi\right) \frac{p_{0}}{p} \\
& \times\left\{\theta\left(E_{p}-p_{0}\right)\left[\mathbf{E}(t)-s^{2} \mathbf{K}(t)\right]+\theta\left(p_{0}-E_{p}\right)\left[E(\alpha, t)-s^{2} F(\alpha, t)-\frac{p}{p_{0}} \sqrt{1-\frac{4 \phi^{2}}{p_{0}^{2}-p^{2}}}\right]\right\},
\end{aligned}
$$

where $s \equiv \sqrt{1-t^{2}}=2 \phi / p_{0}, \alpha \equiv \arcsin \left[p /\left(t p_{0}\right)\right]$, and $F(\alpha, t)$ and $E(\alpha, t)$ are elliptic integrals of the first and second kind,

$$
F(\alpha, t) \equiv \int_{0}^{\alpha} \mathrm{d} x \frac{1}{\sqrt{1-t^{2} \sin ^{2} x}} \quad, \quad E(\alpha, t) \equiv \int_{0}^{\alpha} \mathrm{d} x \sqrt{1-t^{2} \sin ^{2} x},
$$

while $\mathbf{K}(t) \equiv F(\pi / 2, t)$ and $\mathbf{E}(t) \equiv E(\pi / 2, t)$ are complete elliptic integrals of the first and second kind. At $p_{0}=E_{p}, \operatorname{Im} \Pi_{00}^{11}$ is continuous, since then $\alpha=\pi / 2$ and the square root in Eq. (29) vanishes.

It is important to note that the limit $\phi \rightarrow 0$ of the result (29) exists for all $P:$ as $\phi \rightarrow 0, \alpha \rightarrow \arcsin \left(p / p_{0}\right)$ and $t \rightarrow 1$, such that $E(\alpha, t) \rightarrow p / p_{0}$, while $F(\alpha, t) \rightarrow \frac{1}{2} \ln \left[\left(p_{0}+p\right) /\left(p_{0}-p\right)\right]$. Therefore, for $p_{0}>E_{p}$, the imaginary part vanishes like $s^{2} \sim \phi^{2} / p_{0}^{2}$. In the other region, $p_{0} \leq E_{p}$, when $t \rightarrow 1, \mathbf{E}(t) \rightarrow 1$, while $s^{2} \mathbf{K}(t) \rightarrow 0$. In summary, the $\phi \rightarrow 0$ limit of $\operatorname{Im} \Pi_{11}^{00}(P)$ is the imaginary part (22a) of the electric HDL self-energy,

$$
\lim _{\phi \rightarrow 0} \operatorname{Im} \Pi_{11}^{00}(P) \equiv \operatorname{Im} \Pi_{0}^{00}(P) .
$$

The computation of the imaginary part of the magnetic self-energy for gluons of color 1 is completely analogous. With the approximation

$$
\hat{\mathbf{k}}_{1} \cdot \hat{\mathbf{p}} \hat{\mathbf{k}}_{2} \cdot \hat{\mathbf{p}} \simeq(\hat{\mathbf{k}} \cdot \hat{\mathbf{p}})^{2},
$$

valid when $p \ll k \sim \mu$, one derives

$$
\begin{aligned}
\operatorname{Im} \Pi_{11}^{t}(P) \simeq & -\pi \frac{3}{4} m_{g}^{2} \theta\left(p_{0}-2 \phi\right) \frac{p_{0}}{p}\left\{\theta\left(E_{p}-p_{0}\right)\left[\left(1-\frac{p_{0}^{2}}{p^{2}}\right) \mathbf{E}(t)+s^{2} \frac{p_{0}^{2}}{p^{2}} \mathbf{K}(t)\right]\right. \\
& \left.+\theta\left(p_{0}-E_{p}\right)\left[\left(1-\frac{p_{0}^{2}}{p^{2}}\right)\left(E(\alpha, t)-\frac{p}{p_{0}} \sqrt{1-\frac{4 \phi^{2}}{p_{0}^{2}-p^{2}}}\right)+s^{2} \frac{p_{0}^{2}}{p^{2}} F(\alpha, t)\right]\right\} .
\end{aligned}
$$

Again, $\operatorname{Im} \Pi_{11}^{t}$ is continuous at $p_{0}=E_{p}$, and the limit $\phi \rightarrow 0$ exists for all $P$,

$$
\lim _{\phi \rightarrow 0} \operatorname{Im} \Pi_{11}^{t}(P) \equiv \operatorname{Im} \Pi_{0}^{t}(P) .
$$

The computation of the imaginary parts of the electric and magnetic self-energies (20) is straightforward; the final result is

$$
\begin{aligned}
\operatorname{Im} \tilde{\Pi}^{00}(P) \simeq & -\pi \frac{3}{2} m_{g}^{2} \theta\left(p_{0}-2 \phi\right) \frac{p_{0}}{p}\left\{\theta\left(E_{p}-p_{0}\right) \mathbf{E}(t)+\theta\left(p_{0}-E_{p}\right)\left[E(\alpha, t)-\frac{p}{p_{0}} \sqrt{1-\frac{4 \phi^{2}}{p_{0}^{2}-p^{2}}}\right]\right\} \\
\operatorname{Im} \tilde{\Pi}^{t}(P) \simeq & -\pi \frac{3}{4} m_{g}^{2} \theta\left(p_{0}-2 \phi\right) \frac{p_{0}}{p}\left\{\theta\left(E_{p}-p_{0}\right)\left[\left(1-\frac{p_{0}^{2}}{p^{2}}\left(1+s^{2}\right)\right) \mathbf{E}(t)-s^{2}\left(1-2 \frac{p_{0}^{2}}{p^{2}}\right) \mathbf{K}(t)\right]\right. \\
+ & \theta\left(p_{0}-E_{p}\right)\left[\left(1-\frac{p_{0}^{2}}{p^{2}}\left(1+s^{2}\right)\right) E(\alpha, t)-\left(1-\frac{p_{0}^{2}}{p^{2}}\right) \frac{p}{p_{0}} \sqrt{1-\frac{4 \phi^{2}}{p_{0}^{2}-p^{2}}}\right. \\
& \left.\left.-s^{2}\left(1-2 \frac{p_{0}^{2}}{p^{2}}\right) F(\alpha, t)\right]\right\} .
\end{aligned}
$$


As in the previous cases, these functions are continuous at $p_{0}=E_{p}$, and the limit $\phi \rightarrow 0$ exists; together with Eq. (18) one concludes

$$
\begin{aligned}
& \lim _{\phi \rightarrow 0} \operatorname{Im} \Pi_{88}^{00}(P) \equiv \operatorname{Im} \Pi_{0}^{00}(P), \\
& \lim _{\phi \rightarrow 0} \operatorname{Im} \Pi_{88}^{t}(P) \equiv \operatorname{Im} \Pi_{0}^{t}(P) .
\end{aligned}
$$

Figure 2 shows the imaginary parts for a representative value of the gluon momentum $p=4 \phi$ as functions of gluon energy $p_{0}$ (in units of $2 \phi$ ). Comparing the self-energies in a color superconductor (solid lines) with those in a normal conductor (dashed lines) one observes the following two main differences. First, as is obvious from Eqs. (29), (33), (35), and (36), $\operatorname{Im} \Pi_{11}^{00, t}$ and $\operatorname{Im} \tilde{\Pi}^{00, t}$ vanish below $p_{0}=2 \phi$, representing the fact that for such small gluon energies it is impossible to excite quasiparticle-quasihole pairs which would lead to non-vanishing self-energies. For the eighth gluon, however, the functions $\operatorname{Im} \Pi_{88}^{00, t}$ do not vanish because of the admixture of the HDL self-energy, Eq. (18). Second, while the imaginary parts of the HDL self-energies vanish above the light cone $p_{0}=p$, the self-energies in a superconductor are never zero, although they rapidly fall off above $p_{0}=E_{p}$. As will be discussed in more detail in Sec. III, this indicates that, already at one-loop order, gluons are damped on the quasi-particle mass shell [24]. Both features persist for all gluon momenta $p$.

Another interesting property is that the electric self-energy for gluons of color 1 and the magnetic selfenergy for gluons of color 8 are continuous, while the magnetic self-energy for gluons of color 1 has a discontinuity at $p_{0}=2 \phi$ and the electric self-energy for gluons of color 8 has one at $p_{0}=2 \phi$ and one at $p_{0}=p$, the latter arising from the discontinuity of the HDL part in Eq. (18). This leads to characteristic features in the real parts, as will be discussed below. Again, these features are generic for all gluon momenta $p$.

I conclude the discussion noting that for $p_{0} \gg \phi$ the imaginary parts of the gluon self-energies approach the corresponding HDL expressions. They deviate from the latter only for $p_{0} \sim \phi$.

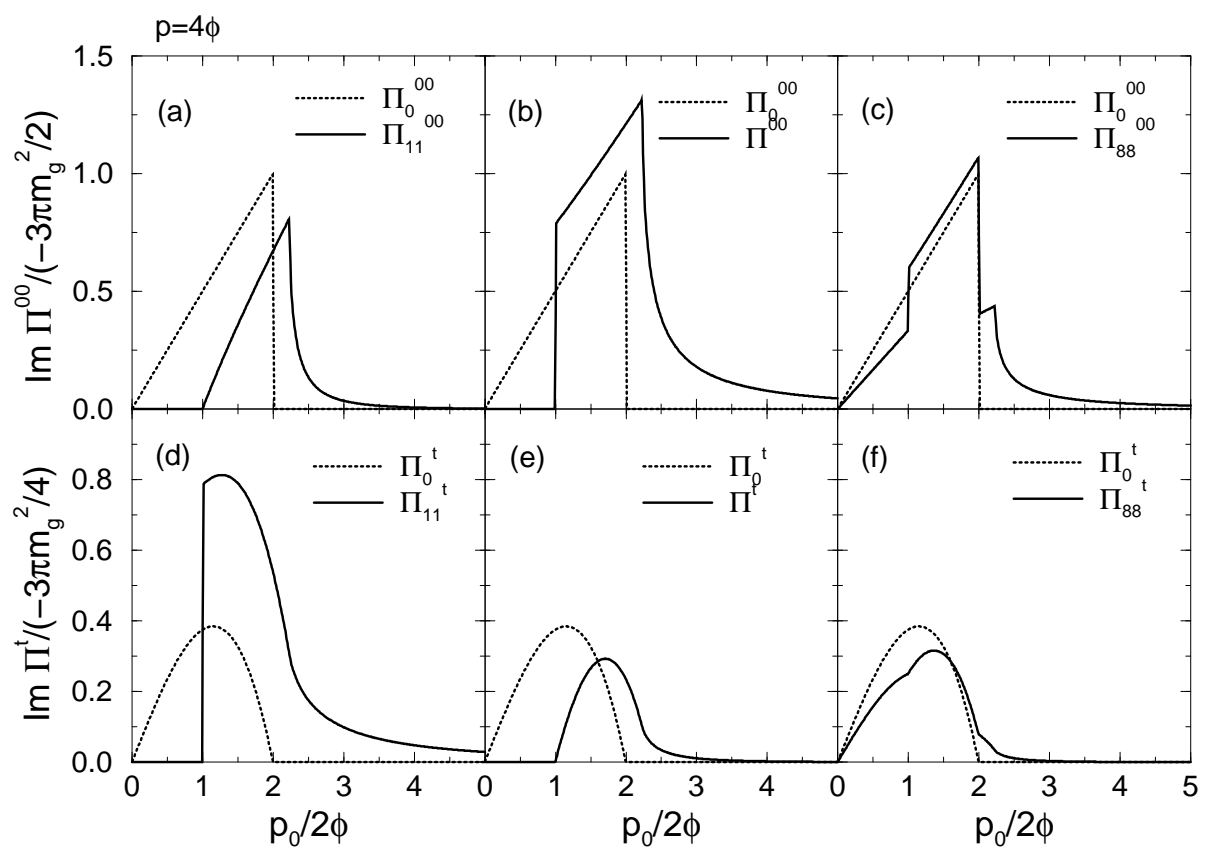

FIG. 2. The imaginary parts of the gluon self-energies as function of energy $p_{0}$ for $p=4 \phi$. Figures (a), (b), and (c) show the electric self-energies, (d), (e), and (f) the magnetic self-energies. The solid lines in (a) and (d) are the imaginary parts of the self-energies for gluons of color 1, in (b) and (e) they are the imaginary parts of the functions $\tilde{\Pi}^{00}$ and $\tilde{\Pi}^{t}$ occurring in the self-energies for gluons of color 8 , the imaginary parts of which are shown in (c) and (f). The dashed lines in all graphs are the imaginary parts of the HDL self-energies. 


\section{B. Real parts}

There are in principle two ways to compute the real parts of the gluon self-energies, either as a principal value integral from Eq. (21), or from the dispersion integral

$$
\operatorname{Re} \Pi\left(p_{0}, \mathbf{p}\right) \equiv \frac{1}{\pi} \mathcal{P} \int_{0}^{\infty} \mathrm{d} \omega \operatorname{Im} \Pi(\omega, \mathbf{p})\left(\frac{1}{\omega+p_{0}}+\frac{1}{\omega-p_{0}}\right)+C,
$$

where $C$ is a (subtraction) constant, and where $\Pi\left(p_{0}, \mathbf{p}\right)$ is assumed to be analytic in the upper complex $p_{0}$-plane.

In the HDL case, Eq. (39) gives for electric gluons

$$
\operatorname{Re} \Pi_{0}^{00}\left(p_{0}, \mathbf{p}\right) \simeq-3 m_{g}^{2}\left(1-\frac{p_{0}}{2 p} \ln \left|\frac{p_{0}+p}{p_{0}-p}\right|\right)+C_{0}^{00},
$$

and for magnetic gluons

$$
\operatorname{Re} \Pi_{0}^{t}\left(p_{0}, \mathbf{p}\right) \simeq \frac{3}{2} m_{g}^{2}\left[\frac{p_{0}^{2}}{p^{2}}+\left(1-\frac{p_{0}^{2}}{p^{2}}\right) \frac{p_{0}}{2 p} \ln \left|\frac{p_{0}+p}{p_{0}-p}\right|-\frac{2}{3}\right]+C_{0}^{t} .
$$

Comparison with a direct calculation via Eq. (19) determines

$$
C_{0}^{00} \equiv 0 \quad, \quad C_{0}^{t} \equiv m_{g}^{2},
$$

such that the term $-2 / 3$ in Eq. (40b) is cancelled by $C_{0}^{t}$ and the magnetic self-energy has the correct zeroenergy limit, $\operatorname{Re} \Pi_{0}^{t}(0, \mathbf{p})=0$, representing the absence of magnetic screening to one-loop order. Thus, the standard expressions for the real parts of the HDL self-energies are recovered [10,23].

It should be remarked that the constant $C_{0}^{t}$ is not a subtraction constant in the mathematical sense. A true subtraction constant would be required if $\operatorname{Im} \Pi_{0}^{t}\left(p_{0}, \mathbf{p}\right)$ did not vanish when $p_{0} \rightarrow \infty$. However, as can be seen from Eq. (22), the imaginary parts of both electric and magnetic HDL self-energy are zero in this limit. The origin of a nonzero constant $C_{0}^{t}$ is purely physical. As discussed in the last subsection, within the present approximations only quasiparticle-quasihole excitations near the Fermi surface give rise to the imaginary part of $\Pi_{a a}^{t}$. This holds also in the normal conducting phase, i.e., for $\operatorname{Im} \Pi_{0}^{t}$. When computing the real part via the dispersion integral (39) these contributions are correctly taken into account. However, what is not accounted for is that, in contrast to the electric self-energies, the real parts of the magnetic self-energies also receive a contribution from quasiparticle-antiparticle excitations, cf. the discussion in Refs. [18, 19. There, I explicitly computed this contribution for $p_{0}=p=0$ in a color superconductor, and found it to be $m_{g}^{2}$, see also [25]26]. As antiparticles are always far from the Fermi surface, it actually does not matter whether one considers these excitations in a superconductor or in a normal conductor, or for nonzero gluon energy and momentum, provided $p_{0}, p \ll \mu$, as is the case here. One therefore concludes that the constant $C_{0}^{t}=m_{g}^{2}$ arises precisely from particle-antiparticle excitations, and that $C_{0}^{00}=0$, because the electric self-energies do not receive such contributions.

For the real parts of the gluon self-energies in a two-flavor color superconductor, I was not able to find expressions in closed form, so that the computation had to be done numerically. One can either directly use Eqs. (17), (20) with Eq. (21), or the dispersion integral (39). It turns out that the second way is numerically far simpler; details can be found in Appendix A. This method does not specify the values of the subtraction constants $C_{a a}^{00}$ and $C_{a a}^{t}, a=1,8$, but from the above discussion on the origin of the result (41), one immediately concludes

$$
C_{11}^{00} \equiv C_{88}^{00} \equiv C_{0}^{00}=0 \quad, \quad C_{11}^{t} \equiv C_{88}^{t} \equiv C_{0}^{t}=m_{g}^{2} .
$$

This has also been confirmed by a direct numerical calculation of the real parts via Eqs. (17), (20) with Eq. (21).

Figure 3 shows the real parts of the gluon self-energies corresponding to the imaginary parts of Fig. 2. The various discontinuities of the imaginary parts discussed above appear in the real parts as logarithmic singularities, due to the relationship between real and imaginary parts dictated by the dispersion integral (39). Consequently, Re $\Pi_{0}^{00}$ has a logarithmic singularity at $p_{0}=p$, cf. Figs. 3 (a), (b), (c), on account of the corresponding discontinuity of the imaginary part, while Re $\Pi_{0}^{t}$ is regular, see Figs. 3 (d), (e), (f). 
By the same line of arguments Re $\Pi_{11}^{00}$, shown in Fig. 3 (a), is regular. The peak at $p_{0} \simeq E_{p}$ visible in this figure is caused by the rapid decrease of the imaginary part in Fig. 2 (a), but it is not a true singularity as long as $\phi$ is nonzero. It becomes a singularity (the same as the one of $\operatorname{Re} \Pi_{0}^{00}$ ) in the limit $\phi \rightarrow 0$. In contrast, $\operatorname{Re} \Pi_{11}^{t}$ has a true logarithmic singularity at $p_{0}=2 \phi$, Fig. 3 (d). This singularity persists for all nonzero $\phi$, but obviously moves towards $p_{0}=0$ as $\phi \rightarrow 0$.

As seen in Fig. 3 (b), the discontinuity of $\operatorname{Im} \tilde{\Pi}^{00}$ at $p_{0}=2 \phi$ causes a singularity in $\operatorname{Re} \tilde{\Pi}^{00}$. Together with the singularity of $\operatorname{Re} \Pi_{0}^{00}$ at $p_{0}=p$, this causes two logarithmic singularities in $\operatorname{Re} \Pi_{88}^{00}$, cf. Fig. 3 (c). As for $\operatorname{Re} \Pi_{11}^{00}$, the peak at $p_{0}=E_{p}$ is not a singularity, but is due to the sharp (but continuous) decrease of $\operatorname{Im} \tilde{\Pi}^{00}$. Only in the limit $\phi \rightarrow 0$, this becomes a true singularity and merges with the one of $\operatorname{Re} \Pi_{0}^{00}$ at $p_{0}=p$. The singularity at $p_{0}=2 \phi$ persists for all nonzero $\phi$. Finally, Re $\tilde{\Pi}^{t}$ is regular, cf. Fig. 3 (e). From Eq. (18) and the regularity of $\operatorname{Re} \Pi_{0}^{t}$ one then concludes that the same holds for $\operatorname{Re} \Pi_{88}^{t}$, Fig. 3 (f).

From the above discussion one concludes that

$$
\begin{aligned}
& \lim _{\phi \rightarrow 0} \operatorname{Re} \Pi_{11}^{00}(P) \equiv \operatorname{Re} \Pi_{0}^{00}(P), \\
& \lim _{\phi \rightarrow 0} \operatorname{Re} \Pi_{88}^{t}(P) \equiv \operatorname{Re} \Pi_{0}^{t}(P) .
\end{aligned}
$$

These equations hold for all $P$ (even for the first equation, if, for $p_{0}=p$, one includes $\infty$ as a possible value for $\left.\lim \operatorname{Re} \Pi_{11}^{00}\right)$.

The functions $\operatorname{Re} \Pi_{11}^{t}$ and $\operatorname{Re} \Pi_{88}^{00}$ also converge towards the corresponding HDL expressions, except in a region of size $\sim \phi$ near the logarithmic singularity at $p_{0}=2 \phi$, where the deviation from the HDL self-energies is large (infinite at $p_{0}=2 \phi$ ). Of course, in the limit $\phi \rightarrow 0$, the point $p_{0}=2 \phi$ moves towards $p_{0}=0$, and the size of the region where there are substantial deviations shrinks to zero. In this sense, the limit $\phi \rightarrow 0$ for $\operatorname{Re} \Pi_{11}^{t}$ and $\operatorname{Re} \Pi_{88}^{00}$ still exists for all $P$ and coincides with the corresponding HDL expressions.

As for the imaginary parts, the real parts of the gluon self-energies in a color superconductor approach the corresponding HDL expressions when $p_{0} \gg \phi$. They deviate from the latter only for $p_{0} \sim \phi$.

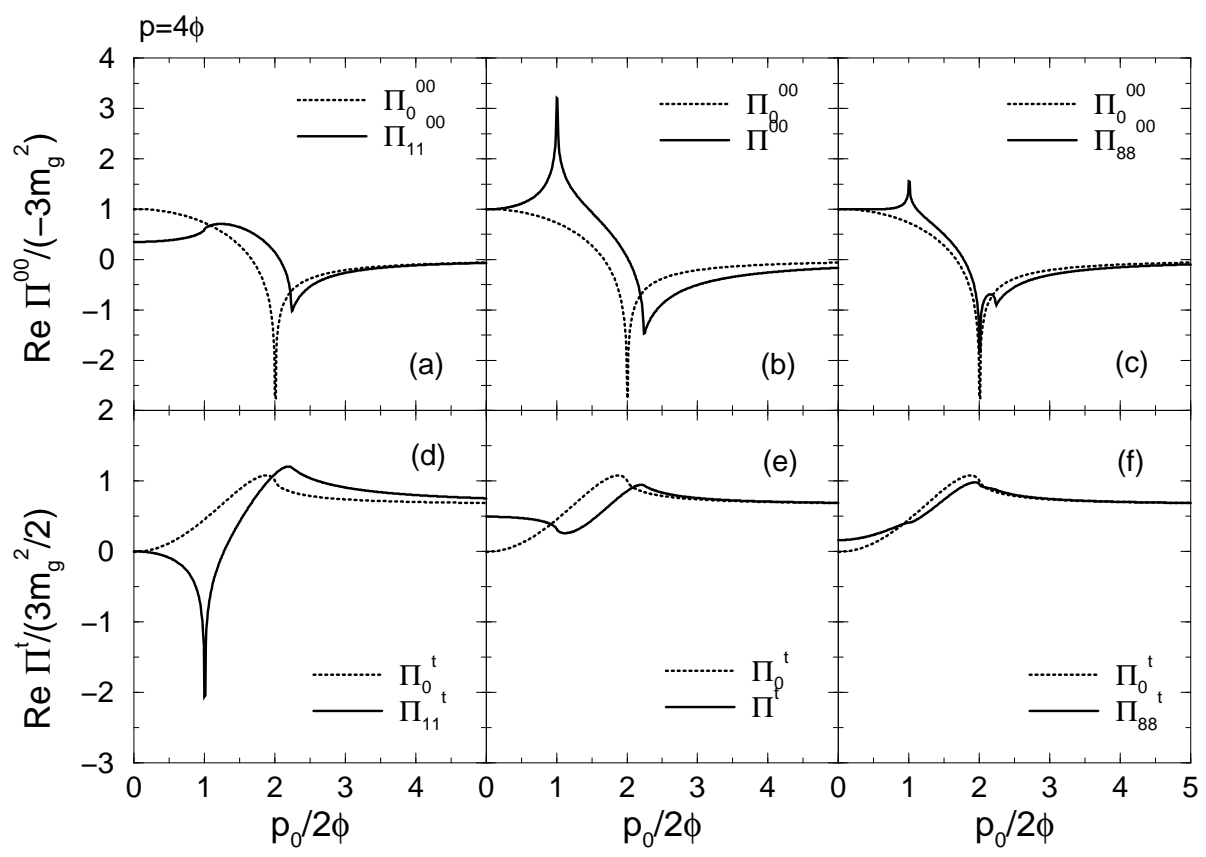

FIG. 3. Same as in Fig. 2, but for the real parts of the gluon self-energies. 


\section{THE SPECTRAL DENSITIES}

The spectral densities are defined by the relations

$$
\rho^{00, t}\left(p_{0}, \mathbf{p}\right) \equiv \frac{1}{\pi} \operatorname{Im} \Delta^{00, t}\left(p_{0}+i \eta, \mathbf{p}\right)
$$

When $\operatorname{Im} \Pi^{00, t}\left(p_{0}, \mathbf{p}\right) \neq 0$, the spectral densities are regular and Eq. (45) is identical to

$$
\begin{aligned}
\rho^{00}\left(p_{0}, \mathbf{p}\right) & =\frac{1}{\pi} \frac{\operatorname{Im} \Pi^{00}\left(p_{0}, \mathbf{p}\right)}{\left[p^{2}-\operatorname{Re} \Pi^{00}\left(p_{0}, \mathbf{p}\right)\right]^{2}+\left[\operatorname{Im} \Pi^{00}\left(p_{0}, \mathbf{p}\right)\right]^{2}}, \\
\rho^{t}\left(p_{0}, \mathbf{p}\right) & =\frac{1}{\pi} \frac{\operatorname{Im} \Pi^{t}\left(p_{0}, \mathbf{p}\right)}{\left[p_{0}^{2}-p^{2}-\operatorname{Re} \Pi^{t}\left(p_{0}, \mathbf{p}\right)\right]^{2}+\left[\operatorname{Im} \Pi^{t}\left(p_{0}, \mathbf{p}\right)\right]^{2}} .
\end{aligned}
$$

When $\operatorname{Im} \Pi^{00, t}\left(p_{0}, \mathbf{p}\right)=0$, for given gluon momentum $\mathbf{p}$ the spectral densities have simple poles, determined by the solution of

$$
\left[p^{2}-\operatorname{Re} \Pi^{00}\left(p_{0}, \mathbf{p}\right)\right]_{p_{0}=\omega^{00}(\mathbf{p})}=0
$$

in the electric case and

$$
\left[p_{0}^{2}-p^{2}-\operatorname{Re} \Pi^{t}\left(p_{0}, \mathbf{p}\right)\right]_{p_{0}=\omega^{t}(\mathbf{p})}=0
$$

in the magnetic case, and are zero elsewhere, i.e.,

$$
\rho^{00, t}\left(p_{0}, \mathbf{p}\right)=-Z^{00, t}(\mathbf{p})\left\{\delta\left[p_{0}-\omega^{00, t}(\mathbf{p})\right]-\delta\left[p_{0}+\omega^{00, t}(\mathbf{p})\right]\right\}
$$

The solutions $\omega^{00, t}(\mathbf{p})$ to Eqs. (47) define the quasiparticle dispersion relations, and

$$
Z^{00, t}(\mathbf{p}) \equiv\left(\left|\frac{\partial\left(\Delta^{00, t}\right)^{-1}\left(p_{0}, \mathbf{p}\right)}{\partial p_{0}}\right|\right)_{p_{0}=\omega^{00, t}(\mathbf{p})}^{-1}
$$

are the residues at the poles $p_{0}=\omega^{00, t}(\mathbf{p})$.

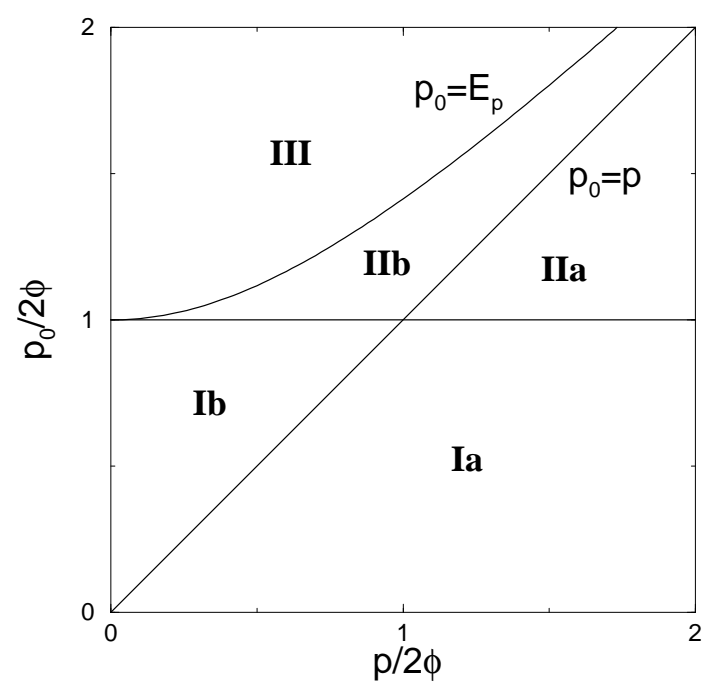

FIG. 4. Schematic plot of the $\left(p_{0}, p\right)$-plane. The HDL spectral density assumes the form (46) in regions Ia and IIa, and the form (48) in regions Ib, IIb, and III. In a two-flavor color superconductor, the spectral density is always of the form (46) in regions IIa, IIb, and III, and of the form (48) in regions Ia and Ib. 
In order to understand the structure of the gluon spectral densities in a two-flavor color superconductor, it is instructive to first remember the structure of the HDL spectral densities in the $\left(p_{0}, p\right)$-plane, cf. Fig. 囵. Below the light cone $p_{0}=p$ (regions Ia and IIa in Fig. (1), the imaginary parts of both electric and magnetic self-energies are nonzero, i.e., gluons are Landau-damped. Therefore, the spectral density is regular and of the form (46). Above the light cone (regions Ib, IIb, and III), the HDL spectral density is of the form (48), with the dispersion relations $p_{0}=\omega_{0}^{00}(\mathbf{p})$ for electric and $p_{0}=\omega_{0}^{t}(\mathbf{p})$ for magnetic gluons. The explicit formulae for the electric (i.e. longitudinal) and magnetic (i.e. transverse) dispersion relations can be found in 10.23]; they are shown graphically in Fig. 5 (dotted curves). In weak coupling, $m_{g} \gg \phi$, and since $\omega_{0}^{00}(0)=\omega_{0}^{t}(0)=m_{g}$, the electric and magnetic dispersion curves lie entirely in region III of Fig. 鳥; in regions Ib and IIb the HDL spectral densities vanish. Figure 6 further clarifies the shape of the HDL spectral densities as functions of $p_{0}$ for an exemplary value of $p$.

On the other hand, in a two-flavor color superconductor, the imaginary parts of the self-energies never vanish for energies $p_{0} \geq 2 \phi$, i.e., in regions IIa, IIb, and III of Fig. 1 . Thus, in these regions the spectral densities are always regular and of the form (46). Nevertheless, for $p_{0} \geq E_{p}$ (region III) the imaginary parts are small, $\operatorname{Im} \Pi\left(p_{0}, \mathbf{p}\right) \sim \phi^{2}$. One can still define dispersion relations $p_{0}=\omega(\mathbf{p})$ through Eqs. (47). These are shown in Fig. . . In weak coupling, $m_{g} \gg \phi$, these dispersion curves are nearly identical to the corresponding HDL dispersion curves, and consequently also lie in region III. (A peculiar feature is the dispersion curve for electric gluons of color 8 , which has a negative slope for small momenta $p \sim \phi$. At present, I cannot exclude that this behavior is merely an artifact caused by neglecting the mixing of these gluons with the excitations of the condensate. This will be clarified in a future publication [27].)

Although the quasiparticles are not stable, like in the HDL case, they decay on a comparatively long time scale $\Gamma^{-1} \sim\left[\operatorname{Im} \Pi\left(p_{0}, \mathbf{p}\right) / p_{0}\right]^{-1} \sim p_{0} / \phi^{2}$. The spectral densities resemble "smeared" delta-functions which peak at $p_{0}=\omega(\mathbf{p})$ and have a width $\sim \operatorname{Im} \Pi\left(p_{0}, \mathbf{p}\right) \sim \phi^{2}$. This can also be seen in Fig. 6, where the electric and magnetic spectral densities in a color superconductor are shown as functions of $p_{0}$ for a fixed value of the gluon momentum, $p=m_{g} / 2$.

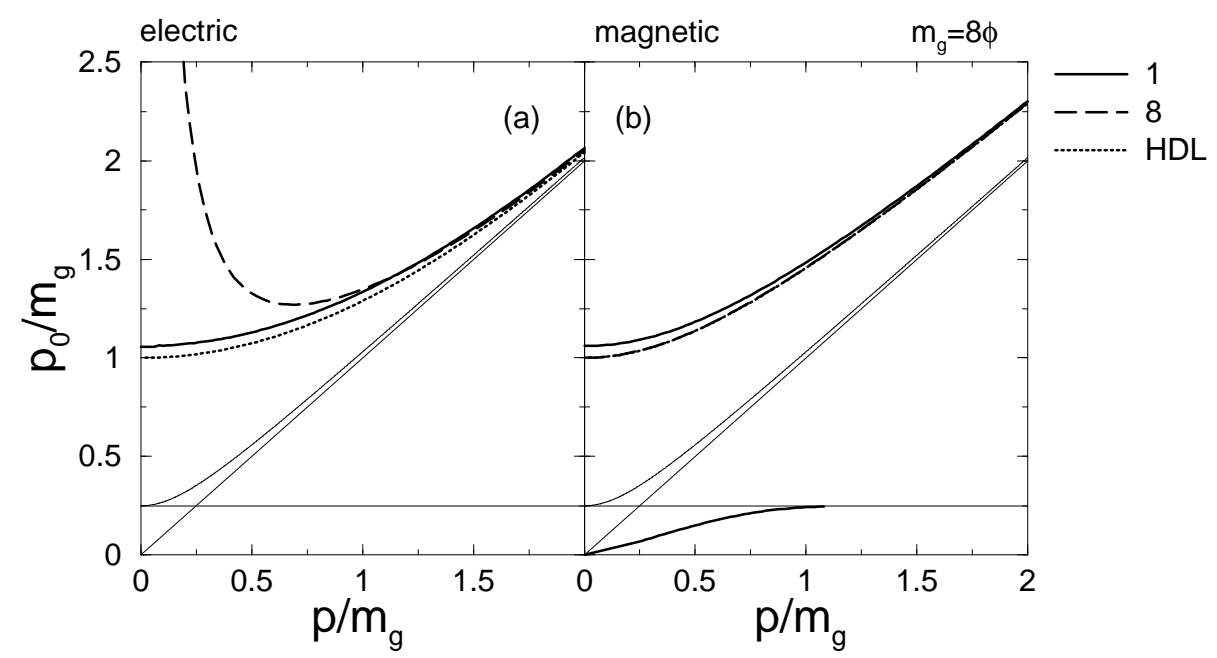

FIG. 5. Dispersion relations for (a) electric and (b) magnetic gluons, $m_{g}=8 \phi$. The different regions of Fig. 14 are indicated by thin solid lines. The dotted curves are the HDL dispersion relations. The solid curves are for gluons of color 1 , the dashed curves for gluons of color 8 . For magnetic gluons, the dispersion curve for gluons of color 8 and the HDL dispersion curve are visually indistinguishable. Note the additional dispersion branch below $p_{0}=2 \phi$ (region Ia of Fig. (4) for magnetic gluons of color 1 . This branch merges with the continuum for $p_{0} \geq 2 \phi$ above $p \simeq 1.1 m_{g}$. 


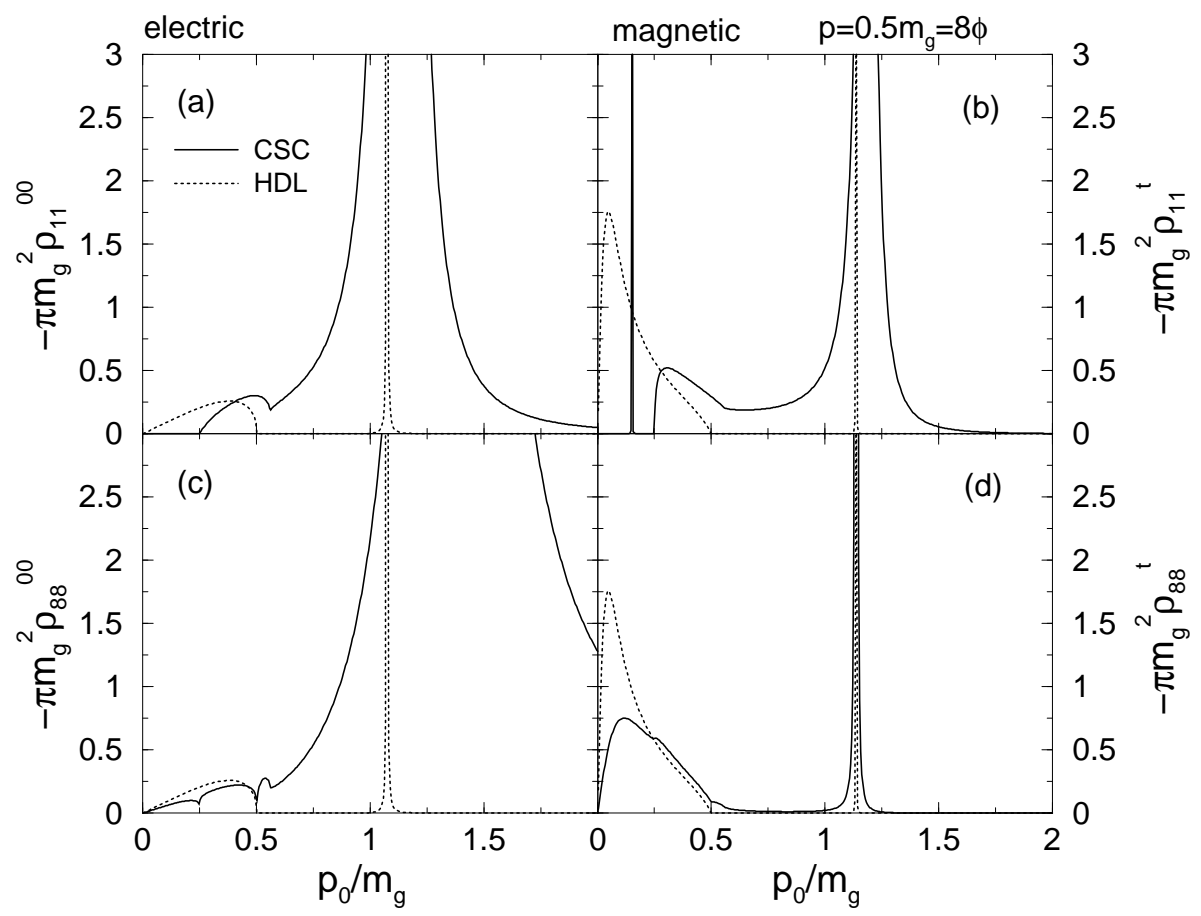

FIG. 6. Electric (a,c) and magnetic (b,d) spectral densities for gluons of color 1 (a,b) and color 8 (c,d), for a fixed value of the gluon momentum, $p=m_{g} / 2$, and $m_{g}=8 \phi$. The dotted curves represent the HDL spectral densities. In order to make the delta-function at the quasiparticle dispersion curves visible, I used the regular form (46) of the spectral density with a numerically small, but nonzero imaginary part.

For $p_{0}<2 \phi$ (regions Ia, Ib in Fig. (4) the imaginary parts of the gluon self-energies in a color superconductor vanish and the spectral densities are of the form (48). It turns out that only magnetic gluons of color 1 have a dispersion branch in this region, cf. Figs. (b) and 6 (b). The origin of this branch, the properties of these gluons, and possible implications for deconfinement in the unbroken $S U(2)_{c}$ sector have been extensively discussed in [21]. Here it is sufficient to note that for $p_{0}, p \ll \phi$, where the dispersion relation is approximately linear, the magnetic self-energy can be written as [21]

$$
\Pi_{11}^{t}(P) \simeq-\frac{m_{g}^{2}}{6 \phi^{2}} p_{0}^{2}
$$

such that the magnetic gluon propagator becomes

$$
\Delta_{11}^{t}\left(p_{0}, \mathbf{p}\right) \simeq \frac{1}{\epsilon p_{0}^{2}-p^{2}},
$$

with the dielectricity constant

$$
\epsilon=1+\frac{m_{g}^{2}}{6 \phi^{2}}
$$

The gluon dispersion relation is

$$
\omega_{11}^{t}(\mathbf{p}) \simeq v p
$$

with the gluon velocity

$$
v \equiv \frac{1}{\sqrt{\epsilon}} \simeq \sqrt{6} \frac{\phi}{m_{g}} \ll 1,
$$

where the approximate equality and the inequality hold in weak coupling. The residue along the dispersion relation is 


$$
Z_{11}^{t}(\mathbf{p}) \simeq \frac{v}{2 p} \simeq \sqrt{\frac{3}{2}} \frac{\phi}{m_{g} p}
$$

For the arguments presented in the next section, it is necessary to consider the limit $\phi \rightarrow 0$ of the spectral densities. In this limit, regions Ia, Ib, and IIb shrink to zero. In region IIa (which becomes the complete region below the light cone $p_{0}=p$ ), the regularity of the self-energies $\Pi_{11}^{00}$ and $\Pi_{88}^{t}$ implies that the spectral densities $\rho_{11}^{00}$ and $\rho_{88}^{t}$ are also regular. Hence, they converge smoothly towards the corresponding HDL expressions,

$$
\begin{aligned}
& \lim _{\phi \rightarrow 0} \rho_{11}^{00}(P)=\rho_{0}^{00}(P) \\
& \lim _{\phi \rightarrow 0} \rho_{88}^{t}(P)=\rho_{0}^{t}(P),
\end{aligned}
$$

for $P$ in region IIa.

As discussed in the previous section, the real parts of the self-energies $\Pi_{11}^{t}$ and $\Pi_{88}^{00}$ also converge towards the corresponding HDL expressions in the limit $\phi \rightarrow 0$, except in a region of size $\sim \phi$ near the logarithmic singularity at $p_{0}=2 \phi$. This singularity is rendered harmless in the spectral densities; the latter simply vanish at this point. The deviation from the HDL spectral densities is, however, still large near this point. The limit $\phi \rightarrow 0$ nevertheless exists, since the point $p_{0}=2 \phi$ merges with the point $p_{0}=0$, where the HDL spectral densities vanish, and the size of the region where there are deviations simply shrinks to zero. Hence also

$$
\begin{aligned}
& \lim _{\phi \rightarrow 0} \rho_{11}^{t}(P)=\rho_{0}^{t}(P), \\
& \lim _{\phi \rightarrow 0} \rho_{88}^{00}(P)=\rho_{0}^{00}(P),
\end{aligned}
$$

for $P$ in region IIa. Finally, in region III the real parts of all self-energies converge smoothly towards the HDL expressions when $\phi \rightarrow 0$. Hence, the dispersion curves in a color superconductor become identical with the HDL dispersion curves. Furthermore, the width of the "smeared" delta-functions in Fig. 6, being $\sim \operatorname{Im} \Pi \sim \phi^{2}$, goes to zero and simultaneously their height becomes infinite: they become true delta-functions, because

$$
\lim _{\delta \rightarrow 0} \frac{\delta / \pi}{x^{2}+\delta^{2}}
$$

is a representation of $\delta(x)$. [To prove this, use Eq. (21).] Thus, Eqs. (56) also hold in region III, and hence in all regions of the $\left(p_{0}, p\right)$-plane which have nonzero measure in the limit $\phi \rightarrow 0$.

\section{THE EFFECT ON THE SOLUTION OF THE GAP EQUATION}

We are now in a position to assess the effect of the modification of the gluon self-energies in a color superconductor on the solution of the gap equation. Let us first perform the Dirac algebra in Eq. (14); details can be found in [3]. After projection onto positive and negative energy, as well as left- and righthanded particle states, and neglecting the contribution from the antiparticle gap, the result for the particle gap function is

$$
\begin{aligned}
\phi^{+}(K) \simeq & \frac{3}{4} g^{2} \frac{T}{V} \sum_{Q} \frac{\phi^{+}(Q)}{q_{0}^{2}-\left[\epsilon_{q}^{+}\left(\phi^{+}\right)\right]^{2}}\left\{\left[\Delta_{11}^{00}(K-Q)-\frac{1}{9} \Delta_{88}^{00}(K-Q)\right] \frac{1+\hat{\mathbf{k}} \cdot \hat{\mathbf{q}}}{2}\right. \\
& \left.+\left[\Delta_{11}^{t}(K-Q)-\frac{1}{9} \Delta_{88}^{t}(K-Q)\right]\left[-\frac{3-\hat{\mathbf{k}} \cdot \hat{\mathbf{q}}}{2}+\frac{1+\hat{\mathbf{k}} \cdot \hat{\mathbf{q}}}{2} \frac{(k-q)^{2}}{(\mathbf{k}-\mathbf{q})^{2}}\right]\right\} .
\end{aligned}
$$

Here, $\epsilon_{q}^{+}\left(\phi^{+}\right) \equiv \sqrt{(q-\mu)^{2}+\left(\phi^{+}\right)^{2}}$, and the chirality index $r, \ell$ of the gap function was omitted, since leftand right-handed gaps decouple and obey the same gap equation. To simplify the notation, in the following I shall also omit the energy index of the gap function, as well as the energy index and the argument of the excitation energy $\epsilon_{q}^{+}\left(\phi^{+}\right)$. 
Now approximate the terms involving $\hat{\mathbf{k}} \cdot \hat{\mathbf{q}}$ as

$$
\frac{1+\hat{\mathbf{k}} \cdot \hat{\mathbf{q}}}{2} \simeq 1 \quad, \quad-\frac{3-\hat{\mathbf{k}} \cdot \hat{\mathbf{q}}}{2}+\frac{1+\hat{\mathbf{k}} \cdot \hat{\mathbf{q}}}{2} \frac{(k-q)^{2}}{(\mathbf{k}-\mathbf{q})^{2}} \simeq-1,
$$

which is appropriate to leading and subleading order in weak coupling. In the resulting equation, add and subtract terms with HDL gluon propagators,

$$
\begin{aligned}
\phi(K) \simeq \frac{2}{3} g^{2} \frac{T}{V} \sum_{Q} \frac{\phi(Q)}{q_{0}^{2}-\epsilon_{q}^{2}}\left[\Delta_{0}^{00}(K-Q)-\Delta_{0}^{t}(K-Q)\right]+\delta \phi(K) \\
\delta \phi(K) \equiv \frac{3}{4} g^{2} \frac{T}{V} \sum_{Q} \frac{\phi(Q)}{q_{0}^{2}-\epsilon_{q}^{2}}\left\{\Delta_{11}^{00}(K-Q)-\Delta_{0}^{00}(K-Q)-\frac{1}{9}\left[\Delta_{88}^{00}(K-Q)-\Delta_{0}^{00}(K-Q)\right]\right. \\
\left.-\left[\Delta_{11}^{t}(K-Q)-\Delta_{0}^{t}(K-Q)\right]+\frac{1}{9}\left[\Delta_{88}^{t}(K-Q)-\Delta_{0}^{t}(K-Q)\right]\right\} .
\end{aligned}
$$

For $\delta \phi(K)=0$, we recover the standard gap equation with the gluon propagators in HDL approximation. To leading and subleading order in weak coupling, this equation is solved by the gap function derived by Son [7]. At the Fermi surface, the value of the gap is given by Eq. (9) with $c$ and $b$ from Eqs. (10) and (12), respectively. The additional term $\delta \phi(K)$ is the correction to this solution. In the following, drawing on the results of the previous sections I determine whether this correction enters at leading order (modifying the value of $c$ ), subleading order (modifying the value of $b$ ), or sub-subleading order (no modification of $c$ and $b$ at all).

First, rewrite the gluon propagators in Eq. (14) in terms of spectral densities, see also Eq. (33) of [3],

$$
\begin{aligned}
\Delta^{00}(P) & \equiv-\frac{1}{p^{2}}+\int_{0}^{1 / T} \mathrm{~d} \tau e^{p_{0} \tau} \int_{0}^{\infty} \mathrm{d} \omega \rho^{00}(\omega, \mathbf{p})\left\{\left[1+n_{B}(\omega / T)\right] e^{-\omega \tau}+n_{B}(\omega / T) e^{\omega \tau}\right\} \\
\Delta^{t}(P) & \equiv \int_{0}^{1 / T} \mathrm{~d} \tau e^{p_{0} \tau} \int_{0}^{\infty} \mathrm{d} \omega \rho^{t}(\omega, \mathbf{p})\left\{\left[1+n_{B}(\omega / T)\right] e^{-\omega \tau}+n_{B}(\omega / T) e^{\omega \tau}\right\}
\end{aligned}
$$

Here, I momentarily reverted to nonzero temperature until the Matsubara sum over fermionic energy $q_{0}=$ $-i(2 n+1) \pi T$ in Eq. (60) has been performed. Consequently, $n_{B}(x) \equiv\left(e^{x}-1\right)^{-1}$ is the Bose-Einstein distribution function. The term $-1 / p^{2}$ in the electric propagator cancels the contribution of $\Delta^{00}(P)$ when $p_{0} \rightarrow \infty[10$. This term is the same for the electric gluon propagators in a superconductor and the electric HDL propagator, because, as we have seen in the previous sections, there is no difference between these propagators for $p_{0} \gg \phi$.

To proceed, one also needs the spectral representation

$$
\frac{\phi(Q)}{q_{0}^{2}-\epsilon_{q}^{2}}=-\int_{0}^{1 / T} \mathrm{~d} \tau e^{q_{0} \tau} \frac{\phi\left(\epsilon_{q}, \mathbf{q}\right)}{2 \epsilon_{q}}\left\{\left[1-n_{F}\left(\epsilon_{q} / T\right)\right] e^{-\epsilon_{q} \tau}-n_{F}\left(\epsilon_{q} / T\right) e^{\epsilon_{q} \tau}\right\},
$$

where $n_{F}(x) \equiv\left(e^{x}+1\right)^{-1}$ is the Fermi-Dirac distribution function. The Matsubara sum over $q_{0}$ can now be performed in the standard manner. After analytic continuation onto the quasiparticle mass shell, $k_{0} \rightarrow \epsilon_{k}+i \eta$, and abbreviating $\phi\left(\epsilon_{q}, \mathbf{q}\right) \equiv \phi_{q}$ the equation for $\delta \phi_{k}$ reads

$$
\begin{aligned}
\delta \phi_{k}=-\frac{3}{4} g^{2} \int \frac{\mathrm{d}^{3} \mathbf{q}}{(2 \pi)^{3}} \frac{\phi_{q}}{2 \epsilon_{q}} \int_{0}^{\infty} \mathrm{d} \omega & {\left[\delta \rho_{11}^{00}(\omega, \mathbf{p})-\frac{1}{9} \delta \rho_{88}^{00}(\omega, \mathbf{p})-\delta \rho_{11}^{t}(\omega, \mathbf{p})+\frac{1}{9} \delta \rho_{88}^{t}(\omega, \mathbf{p})\right] } \\
\times & \left(\frac{1}{\omega+\epsilon_{q}+\epsilon_{k}}+\frac{1}{\omega+\epsilon_{q}-\epsilon_{k}}\right),
\end{aligned}
$$

where $\mathbf{p} \equiv \mathbf{k}-\mathbf{q}$, and where I introduced

$$
\delta \rho_{a a}^{00, t} \equiv \rho_{a a}^{00, t}-\rho_{0}^{00, t} \quad, \quad a=1,8 .
$$

Neglecting a possible imaginary part of the gap function (cf. discussion in [3]), it is implicitly assumed that the energy denominators in Eq. (64) are evaluated with the principal value prescription. 
Finally, note that the spectral densities are isotropic, i.e., they only depend on $|\mathbf{p}| \equiv p$. Substituting the integration variable $\cos \theta \equiv \hat{\mathbf{k}} \cdot \hat{\mathbf{q}}$ by $p$, and interchanging the order in which the $p$ - and $\omega$-integrals are performed, one obtains

$\delta \phi_{k} \simeq \frac{3 g^{2}}{32 \pi^{2}} \int_{\mu-\delta}^{\mu+\delta} \mathrm{d} q \frac{\phi_{q}}{\epsilon_{q}} \int_{0}^{\infty} \mathrm{d} \omega\left\{\mathcal{J}_{11}^{t}(\omega)-\mathcal{J}_{11}^{00}(\omega)-\frac{1}{9}\left[\mathcal{J}_{88}^{t}(\omega)-\mathcal{J}_{88}^{00}(\omega)\right]\right\}\left(\frac{1}{\omega+\epsilon_{q}+\epsilon_{k}}+\frac{1}{\omega+\epsilon_{q}-\epsilon_{k}}\right)$.

where I defined

$$
\mathcal{J}_{a a}^{00, t}(\omega) \equiv \int_{0}^{2 \mu} \mathrm{d} p p \delta \rho_{a a}^{00, t}(\omega, p) .
$$

In deriving Eq. (66) with Eq. (67) I also approximated $k / q \simeq 1, k+q \simeq 2 \mu,|k-q| \simeq 0$, which is valid, since the $q$-integral peaks at the Fermi surface, see also [3]. Moreover, since the gap function $\phi_{q}$ depends strongly on $q$ [7], it is permissible to restrict the momentum integration to a small region around the Fermi surface, $\mu-\delta \leq q \leq \mu+\delta$ where $\delta \ll \mu$.

The important point is that the functions $\mathcal{J}_{a a}^{00, t}$ are dimensionless, i.e., they can only be functions of the dimensionless variables $\omega / m_{g}$ and $\phi / m_{g}$ (or $\omega / \mu$ and $\phi / \mu$; additional factors of $g$ turn out to be of no consequence for the following argument). Let us now estimate these functions.

\section{A. Estimate for $\mathcal{J}_{11}^{00}$}

For $\omega \leq 2 \phi, \rho_{11}^{00} \equiv 0$, and $\delta \rho_{11}^{00}=-\rho_{0}^{00}$. When computing the $p$-integral in $\mathcal{J}_{11}^{00}$, one samples $\rho_{0}^{00}$ in regions Ia and Ib of Fig. 4 . From the explicit form of the spectral density [3]:10,23],

$$
\rho_{0}^{00}(\omega, p)=\theta(p-\omega) \frac{2 M^{2}}{\pi} \frac{\omega}{p}\left\{\left[p^{2}+3 m_{g}^{2}\left(1-\frac{\omega}{2 p} \ln \left|\frac{p+\omega}{p-\omega}\right|\right)\right]^{2}+\left(2 M^{2} \frac{\omega}{p}\right)^{2}\right\}^{-1}
$$

where $M^{2} \equiv 3 \pi m_{g}^{2} / 4$, one deduces that $\rho_{0}^{00}$ vanishes in region Ib, while in region Ia $\rho_{0}^{00}$ peaks for $p$ of order (and slightly larger than) $\omega$, and then falls off $\sim 1 / p^{5}$. Neglecting powers of $g, \mathcal{J}_{11}^{00}$ is at most

$$
\mathcal{J}_{11}^{00}(\omega) \sim \frac{\omega}{m_{g}} \sim \frac{\phi}{m_{g}} .
$$

For $\omega>2 \phi$, the $p$-integral samples regions III, IIb, and IIa. In the latter, both $\rho_{11}^{00}$ and $\rho_{0}^{00}$ are regular, consequently $\delta \rho_{11}^{00}$ is regular, and from the discussion in the previous section one concludes that it vanishes in the limit $\phi \rightarrow 0$. In principle, one could therefore Taylor-expand $\delta \rho_{11}^{00}$ around $\phi=0$. To leading order, one then has in region IIa

$$
\int_{\omega}^{2 \mu} \mathrm{d} p p \delta \rho_{11}^{00}(\omega, p) \sim \frac{\phi}{m_{g}}
$$

In region IIb, $\rho_{0}^{00}=0$, while $\rho_{11}^{00}$ is regular and of order $1 / m_{g}^{2}$. The $p$-integral over this region is therefore at most

$$
\int_{\sqrt{\omega^{2}-4 \phi^{2}}}^{\omega} \mathrm{d} p p \delta \rho_{00}^{11}(\omega, p) \sim \frac{\phi^{2}}{m_{g}^{2}}
$$

Finally, in region III, $\delta \rho_{00}^{11}$ is the difference between a "smeared" delta-function and a true delta-function, which is singular. However, the $p$-integral over $\delta \rho_{00}^{11}$ renders this singularity regular, and since $\delta \rho_{00}^{11}$ vanishes in the limit $\phi \rightarrow 0$ also in this region, the same argument as in region IIa applies,

$$
\int_{0}^{\sqrt{\omega^{2}-4 \phi^{2}}} \mathrm{~d} p p \delta \rho_{00}^{11}(\omega, p) \sim \frac{\phi}{m_{g}} .
$$


Summarizing Eqs. (69) and (70), one concludes

$$
\mathcal{J}_{11}^{00}(\omega) \sim \frac{\phi}{m_{g}}
$$

for all $\omega$.

\section{B. Estimate for $\mathcal{J}_{11}^{t}$}

The estimate for the function $\mathcal{J}_{11}^{t}$ is similar. The difference is that, in region Ia, there is also a contribution from $\rho_{11}^{t}$. Explicitly,

$$
\int_{\omega}^{2 \mu} \mathrm{d} p p \rho_{11}^{t}(\omega, p)=-p\left(\omega_{11}^{t}\right) Z_{11}^{t}(p)\left(\left|\frac{\partial \omega_{11}^{t}(p)}{\partial p}\right|\right)_{p=p\left(\omega_{11}^{t}\right)}^{-1},
$$

where the last term is the Jacobian from the argument of the delta-function in Eq. (48). With Eqs. (53) and (55) one concludes

$$
\int_{\omega}^{2 \mu} \mathrm{d} p p \rho_{11}^{t}(\omega, p) \sim 1
$$

For our order-of-magnitude estimate, it is sufficient to use the approximate form of the magnetic HDL spectral density [3 $[23]$

$$
\rho_{0}^{t}(\omega, p) \simeq \theta(p-\omega) \frac{M^{2}}{\pi} \frac{\omega p}{p^{6}+\left(M^{2} \omega\right)^{2}},
$$

valid for $p \gg \omega$ (this is where the magnetic HDL spectral density has its maximum). One then estimates

$$
\int_{0}^{2 \mu} \mathrm{d} p p \rho_{0}^{t}(\omega, p) \sim \frac{\omega}{m_{g}} \sim \frac{\phi}{m_{g}} .
$$

Thus, to leading order

$$
\mathcal{J}_{11}^{t}(\omega)=\int_{0}^{2 \mu} \mathrm{d} p p \delta \rho_{11}^{t}(\omega, p) \sim 1 .
$$

For $\omega>2 \phi$, the situation is similar as for $\mathcal{J}_{11}^{00}$ and I refrain from repeating the details. One might worry that near $\omega=2 \phi$, where the deviation of $\rho_{11}^{t}$ from $\rho_{0}^{t}$ is large due to the logarithmic singularity of $\operatorname{Re} \Pi_{11}^{t}$, the above arguments do not hold. However, here $\delta \rho_{11}^{t} \simeq-\rho_{0}^{t}$ and with Eq. (74) one convinces oneself that still $\mathcal{J}_{11}^{t} \sim \phi / m_{g}$. In conclusion, to leading order

$$
\mathcal{J}_{11}^{t}(\omega) \sim \theta(2 \phi-\omega) \times 1+\theta(\omega-2 \phi) \times \frac{\phi}{m_{g}} .
$$

\section{Estimate for $\mathcal{J}_{88}^{00}$ and $\mathcal{J}_{88}^{t}$}

The functions $\mathcal{J}_{88}^{00}$ and $\mathcal{J}_{88}^{t}$ are easy to estimate. For $\omega \leq 2 \phi, \delta \rho_{88}^{00, t}$ is the difference between a spectral density with $2 / 3$ of the value of the HDL self-energies and the HDL spectral density itself. Following the same line of arguments as in the previous cases, $\mathcal{J}_{88}^{00, t}(\omega) \sim \phi / m_{g}$. For $\omega>2 \phi$, the situation is similar to that for the functions $\mathcal{J}_{11}^{00, t}$. This immediately leads to the estimate

$$
\mathcal{J}_{88}^{00, t}(\omega) \sim \frac{\phi}{m_{g}}
$$




\section{Estimate for $\delta \phi_{k}$}

We are now in the position to estimate $\delta \phi_{k}$. With the exception of $\mathcal{J}_{11}^{t}$ in the region $\omega \leq 2 \phi$, all functions $\mathcal{J}_{a a}^{00, t}$ contribute at most terms of order $\phi / m_{g}$ to $\delta \phi_{k}$. These contributions are suppressed by $\phi / m_{g}$ relative to the leading or subleading contributions. Additional powers of $g$ are of no consequence, because they are accompanied by at least one power of $\phi$ which is exponentially small in $g, \phi \sim \exp (-1 / g)$. Thus, these contributions cannot change the values of the constants $c$ and $b$ of Eqs. (10) and (12).

The only remaining contribution with a potential impact on the values for $c$ or $b$ is the one from $\mathcal{J}_{11}^{t}$. This contribution gives rise to a $\delta \phi_{k}$ which is of order

$$
\delta \phi_{k} \sim g^{2} \int_{\mu-\delta}^{\mu+\delta} \mathrm{d} q \frac{\phi_{q}}{\epsilon_{q}} \ln \left|\frac{\left(2 \phi+\epsilon_{q}\right)^{2}-\epsilon_{k}^{2}}{\epsilon_{q}^{2}-\epsilon_{k}^{2}}\right| .
$$

The integral can be estimated to be $\sim \phi$, cf. Appendix B, such that $\delta \phi_{k} \sim g^{2} \phi$. This means that $\delta \phi_{k}$ contributes at most to the constant $d$ in Eq. (8), i.e., to sub-subleading order to the solution of the gap equation. Thus, to leading and subleading order, the solution (9) with (10) and (12) is not modified.

\section{SUMMARY, CONCLUSIONS, AND OUTLOOK}

In this paper, I have computed the electric and magnetic self-energies for gluons of adjoint colors 1, 2, 3, and 8 in a color superconductor with two flavors of massless quarks. In an extension of a previous study [18] which was restricted to gluon energies and momenta $p_{0}=p=0$, as well as $p_{0}=0, \phi \ll p \ll \mu$, I now considered the complete range $p_{0}, p \ll \mu$. The results can be qualitatively summarized as follows: the presence of a color-superconducting condensate modifies the gluon self-energies as compared to the selfenergies in a normal-conducting, dense medium only for energies $p_{0} \sim \phi$. For $p_{0} \gg \phi$, the expressions for the self-energies approach the standard hard-dense-loop results.

This study was motivated by the following two observations. First, the weak-coupling result for the color-superconducting gap parameter depends sensitively on the number of unscreened (i.e. massless) gluon degrees of freedom. Second, in a two-flavor color superconductor not only magnetic but also electric gluons are massless in the static, homogeneous limit [18]. This led to the speculation as to whether the modification of the gluon propagator in a color superconductor could change the value of the gap parameter to subleading order in weak coupling, Eq. (9) with (10) and (12). Using the results for the gluon self-energies, I estimated that this is not the case. The physical reason is that the change of gluon properties as compared to those in a normal conductor is limited to the rather small region $p_{0} \leq \phi$.

There remains one unanswered question: the peculiar behavior of the dispersion relation of electric gluons with color 8 displayed in Fig. 同 (a). As discussed above, this could be due to the fact that the present calculation neglects the mixing of this gluon with the excitations of the condensate. This will be addressed in greater detail in a forthcoming publication [27]. Since this phenomena is restricted to momenta $p \sim \phi$, it is, however, unlikely that the conclusions regarding the stability of the value of the gap parameter to leading and subleading order will have to be revised.

\section{ACKNOWLEDGEMENTS}

I am indebted to D. Boyanovsky, G. Carter, C. Manuel, K. Rajagopal, I. Shovkovy, M. Stephanov, D. Son, and especially R. Pisarski for stimulating discussions. I thank J. Knoll for discussions on the contents of Appendix A. G. Carter, R. Pisarski, T. Schäfer, I. Shovkovy, and D. Son are gratefully acknowledged for critically reviewing the manuscript. I thank my colleagues at the RIKEN-BNL Research Center and in the Nuclear and High Energy Theory groups at BNL for their hospitality and friendship extended towards me during the past three and a half years. I also thank RIKEN, BNL, and the U.S. Dept. of Energy for providing the facilities essential for the completion of this work, and Columbia University's Nuclear Theory Group for continuing access to their computing facilities. 


\section{APPENDIX A: NUMERICAL CALCULATION OF THE REAL PART OF THE GLUON SELF-ENERGY}

The real parts of the gluon self-energies can either be computed from Eqs. (17), (20) with Eq. (21), or from the dispersion integral (39). In both cases, this amounts to evaluating a double integral. In the first case, this integral runs over $\xi=k-\mu$ and $x=\cos \theta$, while in the second case, one has to compute elliptic integrals numerically in addition to the integral over $\omega$.

The second way is, however, the simpler one. As the integrand falls off $\sim 1 / \omega^{3}$, one only needs to compute the integral up to some finite value $\Omega$ which is sufficiently large for the required numerical accuracy. One then divides the interval $[0, \Omega]$ into $N$ pieces of size $\delta \Omega=\Omega / N$, such that $\omega_{n}=n \delta \Omega, n=0, \ldots, N$, and computes the integral over $\omega$ piecewise with a generalization of the mean-value theorem as

$$
\operatorname{Re} \Pi\left(p_{0}, \mathbf{p}\right) \simeq \frac{1}{\pi} \sum_{n=0}^{N} \operatorname{Im} \Pi\left(\omega_{n}^{*}, \mathbf{p}\right) \ln \left|\frac{\omega_{n+1}^{2}-p_{0}^{2}}{\omega_{n}^{2}-p_{0}^{2}}\right|+C
$$

with $\omega_{n}^{*} \in\left[\omega_{n}, \omega_{n+1}\right]$ (for practical purposes, $\omega_{n}^{*} \equiv\left(\omega_{n+1}+\omega_{n}\right) / 2$ is a convenient choice). In agreement with the principal-value prescription in (39), one has to make sure to avoid $\omega_{n}=p_{0}$ for any $n$. The advantage of this method is that the imaginary parts can be tabulated prior to the computation of the real parts, such that the computation of the double integral is effectively reduced to the computation of two independent ordinary integrals.

\section{APPENDIX B: ESTIMATE OF THE INTEGRAL IN EQ. (79)}

In this Appendix, I estimate the value of the integral in Eq. (79),

$$
\mathcal{I}\left(\epsilon_{k}\right) \equiv \int_{\mu-\delta}^{\mu+\delta} \mathrm{d} q \frac{\phi_{q}}{\epsilon_{q}} \ln \left|\frac{\left(2 \phi+\epsilon_{q}\right)^{2}-\epsilon_{k}^{2}}{\epsilon_{q}^{2}-\epsilon_{k}^{2}}\right| \text {. }
$$

In order to see whether $\delta \phi_{k}$ contributes to the constants $b, c$, or $d$ in Eq. (8), it is sufficient to perform this estimate at the Fermi surface, $k=\mu$, i.e., $\epsilon_{k}=\phi$. Since the gap function is peaked at the Fermi surface, $\phi_{q} \leq \phi_{\mu} \equiv \phi$

$$
\mathcal{I}(\phi) \leq \phi \int_{\mu-\delta}^{\mu+\delta} \frac{\mathrm{d} q}{\epsilon_{q}} \ln \left[\frac{\left(2 \phi+\epsilon_{q}\right)^{2}-\phi^{2}}{\epsilon_{q}^{2}-\phi^{2}}\right] \equiv 2 \phi \int_{0}^{\delta} \frac{\mathrm{d} \xi}{\epsilon_{q}} \ln \left[\frac{\left(2 \phi+\epsilon_{q}\right)^{2}-\phi^{2}}{\epsilon_{q}^{2}-\phi^{2}}\right],
$$

where $\xi \equiv q-\mu$. Now substitute the integration variable [3]

$$
y \equiv \ln \frac{\xi+\epsilon_{q}}{\phi}
$$

with the result

$$
\mathcal{I}(\phi) \leq 2 \phi \int_{0}^{\ln (2 \delta / \phi)} \mathrm{d} y \ln \left[\frac{\cosh ^{2}(y / 2)+1}{\sinh ^{2}(y / 2)}\right] .
$$

The right-hand side of this equation can be further evaluated,

$$
\mathcal{I}(\phi) \leq 2 \phi\left\{2 \int_{\phi /(2 \delta)}^{1} \frac{\mathrm{d} x}{x} \ln \frac{1+x}{1-x}+\int_{0}^{\ln (2 \delta / \phi)} \mathrm{d} y \ln \left[1+\frac{1}{\cosh ^{2}(y / 2)}\right]\right\}
$$

where $x \equiv e^{-y}$. An upper bound for the first integral is given by extending the lower boundary to zero. The resulting integral can be solved analytically and has the value $\pi^{2} / 4$. An upper bound for the second integral is given by extending the upper boundary to infinity. An integration by parts transforms the resulting integral into 


$$
\mathcal{I}^{\prime} \equiv \int_{0}^{\infty} \mathrm{d} y y \frac{\tanh (y / 2)}{\cosh ^{2}(y / 2)+1}
$$

Substituting $x \equiv \cosh (y / 2)$,

$$
\mathcal{I}^{\prime}=4 \int_{1}^{\infty} \frac{\mathrm{d} x}{x\left(1+x^{2}\right)} \ln \left(x+\sqrt{x^{2}-1}\right) \leq 4 \int_{1}^{\infty} \frac{\mathrm{d} x}{x\left(1+x^{2}\right)} \ln (2 x),
$$

as $\sqrt{x^{2}-1} \leq x$ for $x \in[1, \infty)$. The last integral in (B7) can be solved analytically. Collecting the results, the integral $\mathcal{I}(\phi)$ is bounded from above by

$$
\mathcal{I}(\phi) \leq 2 \phi\left[\frac{7 \pi^{2}}{12}+2(\ln 2)^{2}\right] .
$$

In conclusion, $\mathcal{I}(\phi)$ is of order const. $\times \phi$, and thus $\delta \phi_{k} \sim g^{2} \phi$.

[1] D. Bailin and A. Love, Phys. Rep. 107, 325 (1984).

[2] D.J. Gross and F. Wilczek, Phys. Rev. Lett. 30, 1343 (1973); H.D. Politzer, Phys. Rev. Lett. 30, 1346 (1973).

[3] R.D. Pisarski and D.H. Rischke, Phys. Rev. D 61, 051501, 074017 (2000).

[4] J.R. Schrieffer, Theory of Superconductivity (New York, W.A. Benjamin, 1964).

[5] A.L. Fetter and J.D. Walecka, Quantum Theory of Many-Particle Systems (McGraw-Hill, New York, 1971); A.A. Abrikosov, L.P. Gorkov, and I.E. Dzyaloshinski, Methods of Quantum Field Theory in Statistical Physics (Dover, New York, 1963).

[6] R.D. Pisarski and D.H. Rischke, Phys. Rev. D 60, 094013 (1999).

[7] D.T. Son, Phys. Rev. D 59, 094019 (1999).

[8] T. Schäfer and F. Wilczek, Phys. Rev. D 60, 114033 (1999).

[9] B.C. Barrois, PhD thesis, report UMI 79-04847 (1979).

[10] M. Le Bellac, Thermal Field Theory (Cambridge, Cambridge University Press, 1996).

[11] J.-P. Blaizot and J.-Y. Ollitrault, Phys. Rev. D 48, 1390 (1993); H. Vija and M.H. Thoma, Phys. Lett. B342, 212 (1995); C. Manuel, Phys. Rev. D 53, 5866 (1996).

[12] D.K. Hong, V.A. Miransky, I.A. Shovkovy, and L.C.R. Wijewardhana, Phys. Rev. D 61, 056001 (2000).

[13] S.D.H. Hsu, and M. Schwetz, Nucl. Phys. B572, 211 (2000).

[14] W.E. Brown, J.T. Liu, and H.-C. Ren, Phys. Rev. D 61, 114012 (2000); 62, 054013, 054016 (2000).

[15] S.R. Beane, P.F. Bedaque, and M.J. Savage, Phys. Lett. B483, 131 (2000); nucl-th/0004013.

[16] C. Manuel, Phys. Rev. D 62, 114008 (2000).

[17] K. Rajagopal and E. Shuster, Phys. Rev. D 62, 085007 (2000).

[18] D.H. Rischke, Phys. Rev. D 62, 034007 (2000).

[19] D.H. Rischke, Phys. Rev. D 62, 054017 (2000).

[20] A first attempt to answer this question was made in [N. Evans, J. Hormuzdiar, S.D.H. Hsu, and M. Schwetz, Nucl. Phys. B581, 391 (2000)], where a reduction of the gap was found.

[21] D.H. Rischke, D.T. Son, and M.A. Stephanov, hep-ph/0011379.

[22] D.J. Gross, R.D. Pisarski, and L.G. Yaffe, Rev. Mod. Phys. 53, 43 (1981).

[23] R.D. Pisarski, Physica A 158, 146 (1989).

[24] G.W. Carter and S. Reddy, Phys. Rev. D 62, 103002 (2000).

[25] D.T. Son and M.A. Stephanov, Phys. Rev. D 61, 074012 (2000); 62, 059902 (2000).

[26] D.K. Hong, Phys. Lett. B473, 118 (2000).

[27] I.A. Shovkovy and D.H. Rischke, in preparation. 